VOL. 16-1 (2012), 1-32

\title{
The Axiom of Infinite Choice
}

\author{
Milan R. TASKOvić
}

\begin{abstract}
In this paper we present the Axiom of Infinite Choice: Given any set $P$, there exist at least countable choice functions or there exist at least finite choice functions.

This paper continues the study of the Axiom of Choice by E. Z e r $\mathrm{m}$ e lo [Neuer Beweis für die Möglichkeit einer Wohlordung, Math. Annalen, 65 (1908), 107-128; translated in van Heijenoort 1967, 183-198], and by M. Taskovi ć [The axiom of choice, fixed point theorems, and inductive ordered sets, Proc. Amer. Math. Soc., 116 (1992), 897-904]. Fredholm and Leray-Schauder alternatives are two direct consequences of the Axiom of Infinite Choice!
\end{abstract}

\section{History, origins AND ANNOtATiOns}

We shall first discuss an assumption that appears to be independent of, and yet consistent with, the usual logical assumptions regarding classes and correspondences, but whose absolute validity has been seriously questioned by many authors. This is the so-called Axiom of Choice, which has excited more controversy than any other axiom of set theory since its formulation by Ernst Zermelo in 1908. In this sense, many results are known in the set theory.

In 1904, Zermelo $^{1}$ stated a principle of choice similar to: If $\mathcal{D}$ is a family of nonempty sets, there is a function $f$ such that $f(A) \in A$ for every $A \in \mathcal{D}$; and proved that it implied the well-ordering theorem. In 1908 Zermelo proposed main version of the Axiom of Choice. This is the connection and with a conversations with Erhard Schmidt.

Bertrand Russell in 1906 gave a principle analogous to preceding. He announced this principle as a possible substitute for Zermelo's but he believed

2010 Mathematics Subject Classification. Primary: 01A55, 01A60, 03-03, 03E25, 0403, 04A25; Secondary: 47H10, 05A15, 54H25.

Key words and phrases. The Axiom of Infinite Choice, The Axiom of Choice, Zermelo's Axiom of Choice, Lemma of Infinite Maximality, Zorn's lemma, Restatements of the Axiom of Infinite Choice, Choice functions, Foundation of the Fixed Point Theory, Geometry of the Axiom of Infinite Choice, Axioms of Infinite Choice for Points and Apices.

${ }^{1}$ Before 1904, when $\mathrm{Z}$ e r m e lo published his proof that the axiom of choice implies the well-ordering theorem, the well-ordering theorem was considered as self-evident. $\mathrm{C}$ a n t or and the others used it without hesitation. 
that it was weaker. Zermelo, in 1908 stated and, proved that Russell's and his formulations of the axiom of choice are equivalent. The name "axiom of choice" is due to Zermelo in 1904.

Apparently, the first specific reference to the axiom of choice was given in a paper by G. Peano ${ }^{2}$ in 1890 . In proving an existence theorem for ordinary differential equations, he ran across a situation in which such a statement is needed. In 1886 Peano published a new demonstration of the theorem, due to A. Cauchy, that the differential equation

$$
y^{\prime}=f(x, y), \quad y\left(x_{0}\right)=t_{0},
$$

has a unique solution. Here Peano weakened Cauchy's hypotheses to require only that $f(x, y)$ be continuous. Four years later Peano returned to this theorem and generalized his proof to finite systems of first-order equations.

Beppo Levi in 1902, while discussing the statement that the union of a disjoint set $S$ of nonempty sets has a cardinal number greater than or equal to the cardinal number of $S$, remarked that its proof depended on the possibility of selecting a single member from each element of $S$. Others, including Georg Cantor, had used the principle earlier, but did not mention it specifically.

In 1892 R. Bettazzi, who had just become Peano's colleague at the Military Academy in Turin, published an article on discontinuous real functions - with terminology of infinite many arbitrary choices.

In this time, the Axiom of Choice asserts that for every set $S$ there is a function $f$ which associates each nonempty subset $A$ of $S$ with a unique member $f(A)$ of $A$. From a psychological perspectie, one might express the Axiom by saying that on element is "chosen" from each subset $A$ of $S$. However, if $S$ is infinite, it is difficult to conceive how to make such choices - unless a rule is available to specify an element in each $A$.

\footnotetext{
${ }^{2} \mathrm{G}$ i u s e p p e P e a n o: "But as one cannot apply infinitely many times an arbitrary rule by which one assigns to a class $A$ an individual of this class, a determinate rule is stated her."
} 
David Hilbert, in 1926, once wrote that Zermelo's Axiom of Choice ${ }^{3}$ was the axiom "most attacked up to the present in the mathematical literature..."; to this, Abraham Fraenkel later added that "the axiom of choice is probably the most interesting and, in spite of its late appearance, the most discussed axiom of mathematics, second only to Euclid's axiom of parallels which was introduced more than two thousand years ago."

The equivalence of the axiom of choice and the trichotomy was given by Hartogs in 1915. As in the case of the well-ordering theorem, the trichotomy was considered self-evident and was used without hesitation before 1915 .

As mathematics developed futher there also developed a need for another non-constructive proposition; a principle, which Kuratowski, Hausdorff, Zorn, and others, used to replace transfinite induction and the well-ordering theorem. It appears, at first glance, unrelated to the axiom of choice, but actually is equivalent to it.

This principle and principles similar to it are often referred to as forms of Zorn's lemma. In 1933 Artin and Chevalley first referred to the principle as Zorn's lemma.

The history of maximal principles is quite tangled. The earliest reference to a maximal principle in the literature is in 1907 from Hausdorff.

In 1910 independently Janiszewski, Mazurkiewicz and Zoretti published a special case Hausdorff's principle in the form of a theorem in topology. In 1905 Lindelöf, in 1911 Brouwer, and in 1920 Sierpiński derivated some more general topological theorems from the well-ordering theorem.

In 1922 Kuratowski derived minimal principles equivalent to the preceding principles from the well-ordering theorem. Kuratowski in 1922 used a minimal principle to prove a theorem in analysis, as and R. L. Moore in 1932 .

In set theory, we notice that, all of the usual mathematical concepts can be reduced to the notion of set.

3ZZermelo's Reply to His Critics. During the summer of 1907 Z e r m e lo took stock of the criticisms directed against both his Axiom and his proof of the well-ordering theorem. One in 1908 was a reply to his critics, and the other also in 1908 contained the first axiomatization of set theory. Zermelo's first article in 1908 began with a new demonstration of the well-ordering theorem.

From them he developed the properties of his $\theta$-chains, which generalized Dedekind's earlier concept of chain. Z e r m e l o corresponded with J o u r d a in in 1907, but apparently their letters focused on a generalization of König's theorem.

Although he had read Borel's article and the published correspondence between: $\mathrm{B}$ aire, Borel, $\mathrm{Hadamard}$, and Lebesgue, he concentrated on refuting $\mathrm{P}$ e a n o with whom he had previously feuded over the equivalence theorem.

During 1906 he corresponded with $\mathrm{P}$ o i n c a r é regarding his proof and his axiomatization of set theory. A letter, as well as three others from Poincaré, is kept in Zermelo's Nachlass at the University of Freiburg in Breisgau. De facto, Z e r m e lo emerged as a realist in much situations, perhaps a Platonist!? 
The mathematical concept of a set can be used as the foundation for all known mathematics facts. A flock of pigeons, or a bunch of grapes are examples of sets of things. Sets, as they are usually conceived, have elements or members. An element of a set may be a pigeon, or a grape; i.e., this means an atomistic classical admission sets. It is important to know that a set itself may also be an element of some other set. Mathematics is full of examples of sets of sets.

Within the Cantorian tradition, one can view Zermelo's axiomatization as answering the question: What is a set?! This question has served as a theme in the development of set theory, but one not often discussed openly.

In the meanthime, there has developed a concept of the set-theory damaged school child, so we must ensure that this paradise remains a blooming garden and does not turn into rocky ground and thorny scrub. In this sense, our admission, for this problem in this paper, give a new paradise for set-theory.

We notice that the Axiom of Choice is main spring (origin) for the fixed point theory. In this sense, the fixed point problem for a given mapping $f \mid P$ is very easy to formulate: the question is whether some $\xi \in P$ satisfies $f(\xi)=\xi$. Many problems are reducible to the existence of fixpoints of certain mappings. The question remains whether some statement (of the axiom of choice type) could be equivalently expressed in the fixpoint language as well. The answer is affirmative. In this sense, the equation for $x \in P$ in the following form

$$
\sup \{x, f(x)\}=x \quad\left(\text { or } \sup \left\{x, f^{2}(x)\right\}=x\right)
$$

for a given map $f \mid P$ and for a nonempty partially ordered set $P$ is a key object for new equivalents of the Axiom of Infinite Choice.

In this paper we prove some new equivalents of the Axiom of Infinite Choice in connection with (Eq). These statements are of fixed point type theorems and fixed apex type theorems. Applications in fixed point theory are considered.

Call a poset (=partially ordered set) $P$ inductive (chain complete) when every nonempty chain in $P$ has an upper bound (least upper bound, i.e., supremum) in $P$. Also, call a poset $P$ quasi-inductive (quasi-chain complete) when every nonempty well ordered chain has an upper bound (supremum) in $P$.

Also, we consider the concept of fixed apices for the mapping $f$ of a poset $P$ into itself. A map $f$ of a partially ordered set $P$ to itself has a fixed apex $u \in P$ if for $u \in P$ there is $v \in P$ such that $f(u)=v$ and $f(v)=u$.

Fixed points are clearly fixed apices and the set of all fixed points can be a proper subset of the set of fixed apices.

On the other hand, $f$ has a fixed apex if and only if $f^{2}:=f(f)$ has a fixed point. Indeed, for if $f$ has a fixed apex $u \in P$, then $u=f(v)$ and $v=f(u)$, 
so $f^{2}$ has a fixed point. If the equation $x=f^{2}(x)$ has a solution $\xi=f^{2}(\xi)$ for some $\xi \in P$, then $f$ has fixed apices $\xi, f(\xi) \in P$ because $\xi=f^{2}(\xi)$ and $f(\xi)=f(\xi)$.

An important class of sets is the class of countable sets. More precisely we say that a set $X$ is countable if and only if there is a one-to-one mapping of $\mathbb{N}(:=\{1,2, \ldots, n, \ldots\})$ onto $X$. A set which is not countable is said to be uncountable. ${ }^{4}$

\section{The Lemma of Infinite Maximality}

By the "Axiom of Infinte Choice" we mean a statement in the following form as: Given any set $S$, there exist at least countable choice functions or there exist at least finite choice functions. In this sense we prove some new equivalents of the Axiom of Infinite Choice. But perhaps the most statement equivalent to the Axiom of Infinite Choice is the following statement.

Theorem 1 (Lemma of Infinite Maximality). Let $P$ be an inductive partially ordered set with ordering $\preccurlyeq$, then $P$ has at least countable maximal elements or $P$ has at least finite maximal elements.

Proof. (Application of the Axiom of Infinite Choice). Let card $P=m$ and card $B(m)=\alpha$, where $B(m)$ is denoted the set of all ordinal numbers $\alpha$ such that $\bar{\alpha} \leq m$. For an indirect proof suppose that for every $y \succcurlyeq x$ the set $\{z \in P: y \prec z\}$ is nonempty. Define the transfinite sequences $\left\{y_{\beta}^{k}\right\}_{\beta<\alpha}$ for $k \in \mathbb{N}$ in the following form as

$$
y_{\beta}^{k}=\left\{\begin{array}{l}
\text { the upper bound of }\left\{x_{\gamma}^{k}\right\}_{\gamma<\beta}, \text { if it exist } \\
x \text { otherwise }
\end{array}\right.
$$

for $k \in \mathbb{N}$ and define the transfinte sequences $\left\{x_{\beta}^{k}\right\}_{\beta<\alpha}$ by

$$
x_{\beta}^{k}=f_{k}\left(\left\{z \in X: y_{\beta}^{k} \prec z\right\}\right), \quad \text { for } k \in \mathbb{N},
$$

where $f_{k}:(\mathcal{P}(P) \backslash\{\varnothing\}) \rightarrow P$ for $k \in \mathbb{N}$ are choice functions. Clearly, by (1), $x \preccurlyeq y_{\beta}^{k}$ for every $\beta<\alpha$ and $k \in \mathbb{N}$ so that the set occurring in (2) is nonempty. So the sequences $\left\{x_{\beta}^{k}\right\}_{\beta<\alpha}$ are well defined.

These sequences are increasing. To show this consider the propositional formula $A(\beta)$ for $\beta<\alpha$ meaning: if $\gamma<\xi \leq \beta$ then $x_{\gamma}^{k} \prec x_{\xi}^{k}$ for $k \in \mathbb{N}$. If $A(\gamma)$ for $\gamma<\beta$, then $\left\{x_{\delta}^{k}\right\}_{\delta<\beta}$ are chains, and consequently $x_{\delta}^{k} \leq y_{\beta}^{k}$ for

\footnotetext{
${ }^{4} \mathrm{G}$ e org Cantor: I think of a set as a precipice. On the other hand, L e o p o ld $\mathrm{Kr}$ on e cker brief: Cantor is the corruptor of youth. R i chard D e d e kind: "I think of a set as a closed sack which contains certain specified objects which one doesn't see". D a vi d H i l b e r t in 1925: "No one should ever drive us from the paradise which Cantor created for us". B e r trand R u s s e l: "Thus mathematics may defined as the subject in which we never know that we are talking about, nor whether what we are saying is true".
} 
$\delta<\beta$ and $k \in \mathbb{N}$. Hence, also $x_{\delta}^{k}<x_{\beta}^{k}$ for $\delta<\beta$ and $k \in \mathbb{N}$, i.e., $A(\beta)$ holds. By Principle of Transfinite Induction we have $A(\beta)$ for all $\beta<\alpha$. Now put

$$
M=\bigcup_{\beta<\alpha}\left\{x_{\beta}^{k}\right\} \quad \text { for } k \in \mathbb{N},
$$

then we have $M \subset P$, whence $\operatorname{Card}(M) \leq \operatorname{Card}(P)=m$, whereas $\operatorname{Card}(M)>$ $m$. This shows that for some $\beta<\alpha$ we must have $\left\{z \in P: y_{\beta}^{k} \prec z\right\}=\varnothing$, i.e., $y_{\beta}^{k}(k \in \mathbb{N})$ are maximal elements in $P$, and clearly $x \preccurlyeq y_{\beta}^{k}($ for $k \in \mathbb{N}$ ). The proof is complete.

Theorem 2. Let $P$ be a partially ordered set, then there exist at least countable functions $f_{k}$ (for $k \in \mathbb{N}$ ) or finite functions $f_{k}$ (for $k=1, \ldots, m$ and $a$ fixed number $m \in \mathbb{N}$ ) such that for each nonempty subset $A$ of $P$ is $f_{k}(A) \in A$ for $k \in \mathbb{N}$ or $f_{k}(A) \in A$ for $k=1, \ldots, m$ and a fixed $m \in \mathbb{N}$.

Proof. (Application of the Lemma of Infinite Maximality). Let $\mathcal{A}$ be any collection of nonempty sets, and put $M=\cup \mathcal{A}$. Let $P$ be the family of those sets $F \subset M$ for which the intersection $F \cap A$ contains at most one point for every $A \in \mathcal{A}$. The set $P$ is an ordered set with inclusion $\subset$ and $\varnothing \in P$. If $\mathcal{L} \subset P$ is a chain, then $\cup \mathcal{L} \in P$. In fact, if $\cup \mathcal{L} \cap A$ for an $A \in \mathcal{A}$ contains two different elements, say $x$ and $y$, then there exist sets $D_{x}, D_{y} \in \mathcal{L}$ such that $x \in D_{x} \cap A$ and $y \in D_{y} \cap A$. But since $\mathcal{L}$ is a chain, one of the sets $D_{x}$, $D_{y}$ is contained in the other say $D_{x} \subset D_{y}$. But then $x, y \in D_{y}$ and $D_{y} \cap A$ contains more than one point.

By Theorem 1 there exist in $P$ maximal elements $R_{k}($ for $k \in \mathbb{N}$ ). We will show that $R_{k} \cap A \neq \varnothing$ for every $A \in \mathcal{A}$ and $k \in \mathbb{N}$. If we had $R_{k} \cap A_{0}$ for an $A_{0} \in \mathcal{A}$ and $k \in \mathbb{N}$, then for $x_{0} \in A_{0}$ we might define a set $\mathcal{R}^{*}=R_{k} \cup\left\{x_{0}\right\}$ for $k \in \mathbb{N}$. Clearly, $\mathcal{R}^{*} \in P$ and $\mathcal{R}^{*}$ is larger than $R_{k}$ (for $k \in \mathbb{N}$ ), which is impossible, since $R_{k}$ (for $k \in \mathbb{N}$ ) are maximal elements in $P$. Thus $R_{k} \cap A$ (for $k \in \mathbb{N}$ ) is a singleton for every $A \in \mathcal{A}$ and $k \in \mathbb{N}$, and we can define functions $f_{k}: \mathcal{A} \rightarrow M$ (for $k \in \mathbb{N}$ ) by $f_{k}(A)=A \cap R_{k}$ for $k \in \mathbb{N}$. The functions $f_{k}$ (for $k \in \mathbb{N}$ ) are choice functions.

In the second case, by Theorem 1, there exist in $P$ a finite number maximal elements $R_{0}, R_{1}, \ldots, R_{m}$ (for a fixed $m \in \mathbb{N}$ ). We can define functions $f_{k}: \mathcal{A} \rightarrow M$ (for $\left.k=1, \ldots, m\right)$ by $f_{k}(A)=A \cap R_{k}$ for $k=1, \ldots, m$. Then the functions $f_{k}$ (for $k=1, \ldots, m$ ) are chioice functions. The proof is complete.

\section{Equivalents of the Axiom of Infinite Choice}

In general, equivalents of the Axiom of Infinite Choice appear frequently in almost all branches of mathematics in a large variety of different forms.

In this part of paper we present some equivalent forms of the Axiom of Infinite Choice which are expressible in the following sense. 
Theorem 3. (Axiom of Infinite Choice). Let $S$ be an arbitrary set. Then the following statements are equivalent:

(a) (Axiom of Infinite Choice). Given any set $S$, there exist at least countable functions $f_{k}$ (for $k \in \mathbb{N}$ ) such that for each nonempty subset $A$ of $S$, $f_{k}(A) \in A$ as $k \in \mathbb{N}$ or there exist at least finite choice functions.

(b) If $S$ is a set, $T=\mathcal{P}(S) \backslash\{\varnothing\}$ where $\mathcal{P}$ is the power class, $F$ is the set of all functions from $T$ to $S$ and $g$ is a function from $F$ to $T$, then there exist at least countable functions $f_{k} \in F($ for $k \in \mathbb{N}$ ) or there exist at least finite functions $f_{k} \in F(k=1, \ldots, m)$ such that $f_{k}\left(g\left(f_{k}\right)\right) \in g\left(f_{k}\right)$ for $k \in \mathbb{N}$ or $k=1, \ldots, m$.

Proof. Let (a) holds and let $S$ be a given set. For proof that (a) implies (b) let $S, T=\mathcal{P}(S) \backslash\{\varnothing\}, F$ and $g$ satisfy the hypothesis of (b). Then if $f_{k}$ $(k \in \mathbb{N}$ or $k=1, \ldots, m)$ are choice functions on $T$, then $f_{k} \in F, g\left(f_{k}\right) \in T$ and $f_{k}\left(g\left(f_{k}\right)\right) \in g\left(f_{k}\right)$ for $k \in \mathbb{N}$ or $k=1, \ldots, m$.

This means that (b) is a consequence of (a). Thus, we need only show that (b) implies (a).

Suppose (a) is false. Then there is a set $S$ such that if $T=\mathcal{P}(S) \backslash\{\varnothing\}$, and $F$ is the set of all functions mapping $T$ into $S$ then for all $f_{k} \in F$ (for $k \in \mathbb{N}$ or $k=1, \ldots, m$ ) there is an $U \in T$ such that $f_{k}(U) \notin U$ (for $k \in \mathbb{N}$ or $k=1, \ldots, m)$. Suppose $p \notin S$. For each ordinal $\alpha$ and for each $f_{k} \in F$ (for $k \in \mathbb{N}$ or $k=1, \ldots, m$ ), define

$$
\psi_{f_{k}}(\alpha):= \begin{cases}f_{k}(S \backslash \operatorname{Im} f), & \text { if } f_{k}\left(S \backslash \operatorname{Im} f_{k}\right) \in S \backslash \operatorname{Im} f_{k}, \\ p, & \text { otherwise; }\end{cases}
$$

where $\operatorname{Im} f_{k}$ is image of the mapping $\psi_{f_{k}}(\alpha)$. Since $\psi_{f_{k}}^{-1}$ is a bijection, thus there is an $\alpha$ such that $\psi_{f_{k}}(\alpha)=p$. Let $\alpha_{0}$ be the smallest such $\alpha$. If $\operatorname{Im} f_{k}\left(\alpha_{0}\right)=S$, then $S$ can be well ordered, which implies $T$ has a choice function, contradicting our assumption. Thus, $\operatorname{Im} f_{k}\left(\alpha_{0}\right) \subset S$. Define that $g\left(f_{k}\right)=S \backslash \operatorname{Im} f\left(\alpha_{0}\right)$. Then $g$ is a function with domain $F$, range contained in $T$ and $f_{k}\left(g\left(f_{k}\right)\right) \notin g\left(f_{k}\right)$ for all $f_{k} \in F$ and $k \in \mathbb{N}$ or $k=1, \ldots, m$. This contradicts (b). The proof is complete.

Theorem 4. (Restatements of the Axiom of Infinite Choice). Let $S$ be an arbitrary set and let $\mathcal{D}$ denoted domain and $\mathcal{R}$ denoted range. Then the following statements are equivalent:

(a) (Axiom of Infinite Choice). Given any set $S$, there exist at least countable functions $f_{k}\left(k \in \mathbb{N}\right.$ ) or there is at least finite functions $f_{k}$ ( $k=$ $1, \ldots, m)$ such that for each nonempty subset $A$ of $S, f_{k}(A) \in A$ as $k \in \mathbb{N}$ or $k=1, \ldots, m$.

(b) For every function $f$ there exist countable functions $f_{k}(k \in \mathbb{N})$ or there is at least finite functions $f_{k}(k=1, \ldots, m)$ such that for every $x$, if $x \in \mathcal{D}(f)$ and $f(x) \neq \varnothing$, then $f_{k}(x) \in f(x)$ as $k \in \mathbb{N}$ or $k=1, \ldots, m$. 
(c) For every relation $r$ there exists countable functions $f_{k}(k \in \mathbb{N})$ or there is at least finite functions $f_{k}(k=1, \ldots, m)$ such that $\mathcal{D}\left(f_{k}\right)=\mathcal{D}(r)$ and $f_{k} \subset r$ as $k \in \mathbb{N}$. or $k=1, \ldots, m$.

(d) For every function $f$ there exist countable functions $f_{k}(k \in \mathbb{N})$ or there is at least finite functions $f_{k}(k=1, \ldots, m)$ such that $\mathcal{D}\left(f_{k}\right)=\mathcal{R}(f)$ and for every $x \in \mathcal{D}\left(f_{k}\right)$ is $f\left(f_{k}(x)\right)=x$ as $k \in \mathbb{N}$ or $k=1, \ldots, m$.

Proof. We shall first show that (a) implies (b). Let $f$ be an arbitrary function. Let $S=\mathcal{R}(f)$ and let $F_{k}(k \in \mathbb{N}$ or $k=1, \ldots, m)$ be choice functions on $S$. Define functions $f_{k}(k \in \mathbb{N}$ or $k=1, \ldots, m)$ such that for each $x \in \mathcal{D}(f), f_{k}(x)=F_{k}(f(x))$. Then $f_{k}(k \in \mathbb{N}$ or $k=1, \ldots, m)$ are the required functions.

Also, (b) implies (a). In this sense, let $\mathcal{L}$ be a set of nonempty sets of $S$. Let $f$ be a bijection function such that $\mathcal{R}(f)=\mathcal{L}$. Define countable functions $F_{k}(k \in \mathbb{N}$ or $k=1, \ldots, m)$ such that for each $x \in \mathcal{L}$ we have $F_{k}(x)=f_{k}\left(f^{-1}(x)\right)$, where $f_{k}(k \in \mathbb{N}$ or $k=1, \ldots, m$ or $k=1, \ldots, m)$ are defined by (b). Then $F_{k}(k \in \mathbb{N}$ or $k=1, \ldots, m)$ are the required choice functions.

For proof that (c) implies (d), let $f$ be an arbitrary function and let $r=\{(x, y):(y, x) \in f\}$. Then, (c) implies that there exist functions $f_{k}$ $(k \in \mathbb{N}$ or $k=1, \ldots, m)$ such that $\mathcal{D}\left(f_{k}\right)=\mathcal{D}(r)$ and $f_{k} \subset r$ as $k \in \mathbb{N}$ or $k=1, \ldots, m$. Clearly, for every $x \in \mathcal{R}(f)=\mathcal{R}\left(f_{k}\right)$ we have $f\left(f_{k}(x)\right)=x$ as $k \in \mathbb{N}$ or $k=1, \ldots, m$.

Also, (d) implies (c). Indeed, let $r$ be an arbitrary relation and define a function $h$ as follows that $h=\{((x, y), x):(x, y) \in r\}$. Then (d) implies that there exist functions $F_{k}(k \in \mathbb{N}$ or $k=1, \ldots, m)$ such that $\mathcal{D}\left(F_{k}\right)=\mathcal{D}(h)$ and for every $x \in \mathcal{D}\left(F_{k}\right)$ we have $h\left(F_{k}(x)\right)=x$ as $k \in \mathbb{N}$ or $k=1, \ldots, m$. Now, $F_{k}(k \in \mathbb{N}$ or $k=1, \ldots, m)$ are ordered pairs, so we define $f_{k}(x)$ as $k \in \mathbb{N}$ or $k=1, \ldots, m$ to be the second coordinate of $F_{k}(k \in \mathbb{N}$ or $k=1, \ldots, m)$ for each $x \in \mathcal{D}\left(F_{k}\right)=\mathcal{D}(r)$ as $k \in \mathbb{N}$ or $k=1, \ldots, m$. Clearly, $\mathcal{D}\left(f_{k}\right)=\mathcal{D}(r), f_{k}(k \in \mathbb{N}$ or $k=1, \ldots, m)$ are functions, and $f_{k} \subset r$ as $k \in \mathbb{N}$ or $k=1, \ldots, m$.

In connection with this, (c) implies (b), also. Indeed, let $f$ be an arbitrary function. Define a relation $r$ as follows: $r=\{(x, y): y \in f(x)\}$. Then, (c) implies that there exist functions $f_{k}(k \in \mathbb{N}$ or $k=1, \ldots, m)$ such that $\mathcal{D}\left(f_{k}\right)=\mathcal{D}(r)$ and $f_{k} \subset r$ as $k \in \mathbb{N}$ or $k=1, \ldots, m$. In this case, $f_{k}(k \in \mathbb{N}$ or $k=1, \ldots, m)$ are the required functions.

Also, (b) implies (c). In this sense, let $r$ be an arbitrary relation. Define a function $h$ as follows: $h(x)=\{y:(x, y) \in r\}$ for $x \in \mathcal{D}(r)$. Also, (b) implies that there exist functions $f_{k}(k \in \mathbb{N}$ or $k=1, \ldots, m)$ such that if $x \in \mathcal{D}(h)$ and $h(x) \neq \varnothing$, then $f_{k}(x) \in h(x)$ as $k \in \mathbb{N}$ or $k=1, \ldots, m$. Then, $f_{k}(k \in \mathbb{N}$ or $k=1, \ldots, m)$ are the required functions. Now, the proof is complete.

In connection with the preceding facts, research continued on the deductive strength of various statements relative to the Boolean Prime Ideal 
Theorem. In 1961 Jan Mycielski considered the following statement $P_{n}$ for each $n>1$ in form: If $G$ is a graph such that every finite subgraph of $G$ can be colored with $n$ colors, then $G$ itself can be colored with $n$ colors. Höft and Howard later, in 1973, gave a graph-theoretic equivalent of the Axiom of Choice.

In 1955 W. Kinna and K. Wagner, introduced a different kind of proposition as a weakening of the Axiom of Choice: For every set $S$ there is a function $f$ such that, for each subset $A$ of $S$ with two or more elements, $f(A)$ is a nonempty proper subset of $A$.

Also, in $1962 \mathrm{~A}$. Levy considered the following propositions $Z(n)$, closely related to the Kinna-Wagner Principle in form: For every family $\mathcal{F}$ of nonempty sets there is a function $f$ such that, for each $A$ in $\mathcal{F}, f(A)$, is a nonempty subset of $A$ having at most $n$ elements.

In connection with the preceding facts for the Axiom of Infinite Choice we have the following result which is a consequence of the preceding two statements.

Theorem 5. (Axiom of Infinite Choice). Let $S$ be an arbitrary set. Then the following statements are equivalent:

(a) Let $m \geq 1$ be a natural number. For every set $S$ there exists an ordinal number $\alpha$ and countable functions $f_{k}(k \in \mathbb{N})$ or finite functions $f_{k}(k=1, \ldots, s)$ defined on $\alpha$ such that $f_{k}(\beta) \preccurlyeq m$ for every $\beta<\alpha$ and $\cup_{\beta<\alpha} f_{k}(\beta)=S$ as $k \in \mathbb{N}$ or $k=1, \ldots, s$.

(b) For every set $S$ there exist a natural number $m \geq 1$, an ordinal number $\alpha$, and countable functions $f_{k}(k \in \mathbb{N})$ or finite functions $f_{k}(k=1, \ldots, s)$ defined on $\alpha$ such that $f_{k}(\beta) \preccurlyeq m$ for every $\beta<\alpha$ and $\cup_{\beta<\alpha} f_{k}(\beta)=S$ as $k \in \mathbb{N}$ or $k=1, \ldots, s$.

(c) Let $m \geq 1$ be a natural number. If $\mathcal{F}$ is a set of nonempty sets, then there exist countable functions $f_{k}(k \in \mathbb{N})$ or finite functions $f_{k}(k=1, \ldots, s)$ such that for each $A \in \mathcal{F}, f_{k}(A)$ is a nonempty subset of $A$ with $f_{k}(A) \preccurlyeq m$ as $k \in \mathbb{N}$ or $k=1, \ldots, s$.

(d) If $\mathcal{F}$ is a set of nonempty sets, then there exist a natural number $m \geq 1$ and countable functions $f_{k}(k \in \mathbb{N})$ or finite functions $f_{k}(k=1, \ldots, s)$ such that for each $A \in \mathcal{F}, f_{k}(A)$ is a nonempty subset of $A$ with $f_{k}(A) \preccurlyeq m$ as $k \in \mathbb{N}$ or $k=1, \ldots, s$.

We notice that the following implications are clear and a totally analogy with the preceding proofs: (a) is equivalent to the (b). Also, (c) and (d) are consequences of (b). Thus, we need only show that (d) implies (b).

In this sense, let $A$ be a set and let $\mathcal{F}$ be the set of all nonempty subsets of $A$. By (d), there exist a natural number $m \geq 1$ and countable or finite functions $F_{k}(k \in \mathbb{N}$ or $k=1, \ldots, s)$ such that for each $A \in \mathcal{F}, F_{k}(A) \neq \varnothing$, $F_{k}(A) \subset A$, and $F_{k}(A) \preccurlyeq m$ for $k \in \mathbb{N}$ or $k=1, \ldots, s$.

Define $F_{k}(\varnothing)=u$, where $u \notin A$. Define the functions $G_{k}(k \in \mathbb{N}$ or $k=$ $1, \ldots, s)$ as follows: For all ordinal numbers $\alpha, G_{k}(\alpha)=F_{k}\left(A \backslash \cup_{\beta<\alpha} G_{k}(\beta)\right)$. 
Now, we have that $G_{k}^{-1}$ is bijective on $\mathcal{R}\left(G_{k}\right) \cap \mathcal{R}(A)$, and there is an ordinal $\alpha$ such that $\cup \operatorname{Im} G_{k}(\alpha)=A$. Then define $f_{k}=G_{k} \mid \alpha$, and we have $m \geq 1$ is the natural number, $\alpha$ is the ordinal number and $f_{k}(k \in \mathbb{N}$ or $k=1, \ldots, s)$ are the functions which satisfies (b). The proof is complete.

It wasn't until 1935 that Max Zorn ${ }^{5}$ published his paper. He was the first one to use a maximal principle in algebra. He stated without proof that this the maximal principle is equivalent to the axiom of choice. For this proof Zorn credits Artin and Kuratowski.

In France, where the Axiom had been so poorly received three decades earlier, Zorn's friend Chevalley introduced the maximum principle to the Bourbakists and after dedicing, Bourbaki stated Zorn's principle as a corollary.

In 1940, also influenced by Zorn, the Princeton topologist John Tukey deduced from the Axiom four variants of what he termed Zorn's lemma, and sketched a proof of their equivalence to the Axiom of Choice.

Nevertheless, there remained one final independent rediscovery, due to the German algebraist O. Teichmüller then in 1939 a Privatdozent at Berlin. This principle is often referred to as form of Teichmüller-Tukey lemma.

The Serbian mathematician Djuro Kurepa found in 1952 a number of relations $R$ such that the corresponding maximal principle was an equivalent.

In 1960 two American mathematicians, Herman and Jean Rubin, were prompted by Kurepa's research to consider maximal principles. In addition, $H$. Rubin found two statements which were equivalent to the Axiom of Choice in $Z F$, but were weaker in $Z F U$. In 1963 the Rubins published a book summarizing and completing much of the earlier work on equivalents.

On the other hand, in 1936 the American mathematician Marshall Stone, then at Harvard, contributed his influential findings on the representation of Boolean rings. Stone deduced a proposition equivalent to it and later known as the Stone Representation Theorem.

In 1939 A. Tarski was studying the number of prime ideals found in rings of sets. Later, in 1940, Birkohoff observed that his representation theorem for distributive lattices had been inspired by the researches of Tarski.

Probably the most well-known and important topological equivalent of the Axiom of Choice is the Tychonoff Compactness Theorem in 1935 from a maximal principle for which in $1955 \mathrm{~J}$. Kelley proved the converse.

\footnotetext{
${ }^{5}$ What were the beginnings of Zorn's principle? According to his later reminiscences, he first formulated it at Hamburg in 1933, where Claude Chevalley and Emil Artin then took it up as well. Indeed, when $\mathrm{Z}$ o r n applied it to obtain representatives from certain equivalence classes on a group, A r t i n recognized that Zorn's principle yields the Axiom of Choice. By late in 1934, Zorn's principle had found users in the United States who dubbed in Zorn's lemma. In October, when Zorn lectured on his principle to the American Mathematical Society in New York, S ol o m on L e f s chetz recomended that Zorn publish his result. The paper appeared, the following year, in 1935.
} 
The second development occured on the frontier between algebra, analysis, and set theory: Stefan Banach's researches at Lwów on functional analysis.

In 1929 Banach established a fundamental result later known as the HahnBanach theorem. To obtain this result, Banach relied on the well-ordering theorem. In this sense, in analysis, the following facts are connection and hold: Krein-Milman theorem, Alaoglu's theorem, and Bell-Fremlin theorem, as and many others.

The fixpoint problem for a given mapping $f \mid P$ is very easy to formulate: the question is whether some $\zeta \in P$ satisfies $f(\zeta)=\zeta$. Many problems are reducible to the existence of fixpoints of certain mappings. The question remains whether statement (of the Axiom of Choice type) could be equivalently expressed in the fixpoint language as well. The answer is affirmative.

What Hilbert had in mind was a new postulate for logic. Since he considered this postulate to go beyond a finitistic logic, in 1923 he named in the Transfinite Axiom:

$$
A\left(\tau_{x}(A(x))\right) \rightarrow A(y) ;
$$

in effect it asserted that, if a proposition $A(c)$ is provable, where $c$ is the value given by the function $\tau$ operating on the formula $A(x)$, then $A(y)$ is provable for every value of $y$. Thus $\tau$ selected a value $c$ for which $A(c)$ would be unprovable of this could occur at all.

When Hilbert returned to his Transfinite Axiom in 1926, he reformulated it in such a way that it more nearly paralleled the Axiom of Choice:

$$
A(y) \rightarrow A\left(\varepsilon_{x}(A(x))\right) ;
$$

this $\varepsilon$-axiom as he later called it, stated in effect that if $A(c)$ held for some value $c$, then $\varepsilon_{x}(A(x))$ was one such $c$.

An existence theorem asserts the existence of an object belonging to a certain set and possessing certain properties. Many existence theorems can be formulated so that the under lying set is a partially ordered set and the crucial property is maximality.

This principle and principles similar to it are often referred to as a form of Zorn's lemma. A strong form of Zorn's lemma is due to Bourbaki. In this paper we present a new strong form of Zorn's and Bourbaki's lemma. On the other hand, we notice that a statement on the existence of maximal elements (in certain partially ordered complete subsets of a normed linear space) played a central role in the proof of the fundamental statement of Bishop and Phelps on the density of the set of support points of a closed convex subset of a Banach space.

The transfinite induction argument is based on Zorn's lemma. This argument was later used in a different setting by Brøndsted and Rockafellar, Browder, Ekeland, Brøndsted and others. Recently Brézis and Browder 
proved a very general principle concerning order relations which unifies a number of diverse results in nonlinear functional analysis.

In connection with the preceding facts we have the following results as new equivalents of the Axiom of Infinite Choice.

Theorem 6. (Lemma of Infinite Maximality). Let $P:=(P, \preccurlyeq)$ be a partially ordered set. Then the following statements are equivalent:

(a) (Lemma of Infinite Maximality). Let $P$ be an inductive poset, i.e., every nonempty ordered chain in $P$ has an upper bound in $P$, then $P$ has countable maximal elements or $P$ has finite maximal elements.

(b) (Lemma of Infinite Maximality). Let $P$ be a quasi inductive poset, i.e., every nonempty well ordered chain in $P$ has an upper bound in $P$, then $P$ has at least countable maximal elements or $P$ has finite maximal elements.

(c) Let $P$ be a quasi inductive partially ordered set and $f$ a mapping from $P$ into $P$ such that the following fact holds in the form as

$$
x \preccurlyeq f(x) \text { for all } x \in \operatorname{Sub} f(P),
$$

where $\operatorname{Sub} f(P):=f(P) \cup\{a \in P \mid a=u b C$ for some chain in $f(P)\}$ and where $u b C$ is an upper bound of $C$. Then $f$ has at least countable or finite fixed points.

(d) Let $P$ be a chain complete partially ordered set, i.e., every nonempty chain in $P$ has a least upper bound in $P$, and $f$ a mapping from $P$ into itself such that: (i) there is an element $\theta \in P$ with $\theta \preccurlyeq x$ for all $x \in P$, (ii) $x \preccurlyeq f(x)$ for all $x \in P$, (iii) if $x, y \in P$ and $x \preccurlyeq y \preccurlyeq f(x)$ then either $x=y$ or $f(x) \preccurlyeq f(y)$. Then there exist $\xi_{k} \in P$ with $f\left(\xi_{k}\right)=\xi_{k}$ for all $k \in \mathbb{N}$ or $f\left(\xi_{k}\right)=\xi_{k}$ for finite $k=1, \ldots, m$.

Proof. We notice that from the former facts (see: Tasković [23]), (a) is equivalent to (b).

Also, (b) implies (c). Indeed, by (b) there exist countable or finite maximal elements of $P$. Let $z_{k} \in P$ for $k \in \mathbb{N}$ or $k=1, \ldots, m$ maximal elements, i.e., Sub $f(P)$ has maximal elements $z_{k} \in \operatorname{Sub} f(P)$ for $k \in \mathbb{N}$ or $k=1, \ldots, m$. From condition (M) we have $z_{k} \preccurlyeq f\left(z_{k}\right)$ and, because $z_{k}$ are maximal elements of the set $\operatorname{Sub} f(P), f\left(z_{k}\right) \preccurlyeq z_{k}$ for $k \in \mathbb{N}$ or $k=1, \ldots, m$. Hence, $f\left(z_{k}\right)=z_{k}$ for $k \in \mathbb{N}$ or $k=1, \ldots, m$, so, $f$ has countable or finite fixed points in $P$. In the second case, by (b) there exist in $P$ a finite number maximal elements, i.e., $f$ has finite fixed points in $P$. This means that (c) holds. Applying (c) to the set Sub $f(P)$, we obtain that $f$ has countable or $f$ has finite fixed points, i.e., we obtain that (d) holds.

Thus, we need only show that (d) implies (a), i.e., that (d) implies Lemma of Maximality. In this sense, suppose that the result (a) is false.

Then for each $x \in P$ there exists $y \in P$ with $x \preccurlyeq y$ and $x \neq y$. Let $\mathcal{T}_{0}$ be the family of all nonempty chains of $P$ and let $\mathcal{T}=\mathcal{T}_{0} \cup\{\varnothing\}$. The family $\mathcal{T}$ is partially ordered by the inclusion relation between subsets of $P$. For each 
$A \in \mathcal{T}_{0}$ the set

$$
U_{A}=\{x \in P: x \quad \text { is an upper bound for } A \text { and } x \notin A\}
$$

is nonempty because, if $x$ is an upper bound for $A$ and $y \in P$ is such that $x \preccurlyeq y$ and $x \neq y$, then $y \in U_{A}$. Let $U_{\varnothing}=\left\{x_{0}\right\}$, where $x_{0}$ is an arbitrary element of $P$. Let $g$ be a mapping with domain $X:=\left\{U_{A}: A \in \mathcal{T}\right\}$, and now, we define a mapping $g$ from $X$ into itself by $g(x)=x$, i.e., $g$ is the identity mapping. For each $A \in \mathcal{T}$ let $f(A)=A \cup\left\{g\left(U_{A}\right)\right\}$. By definition of $g$ and $U_{A}$ we have $a \preccurlyeq g\left(U_{A}\right)$ for all $a \in A$ and all $A \in \mathcal{T}_{0}$. It is now clear that $f(A) \in \mathcal{T}$ for all $A \in \mathcal{T}$ and hence $f$ maps $\mathcal{T}$ into itself.

We shall prove that $\mathcal{T}$, partially ordered by inclusion, and $f$ satisfy the conditions of (d). First we observe that $\varnothing \in \mathcal{T}$ and $\varnothing \in A$ for all $A \in \mathcal{T}$ so $\mathcal{T}$ satisfies condition (i) of (d). Next let $\mathcal{R}$ be a nonempty subfamily of $\mathcal{T}$ such that $\mathcal{R}$ is chain ordered by inclusion and let $A=\cup_{B \in \mathcal{R}} B$. Let $a, b \in A$. There are sets $C, D \in \mathcal{R}$ with $a \in C$ and $b \in D$. Since $\mathcal{R}$ is a chain ordered by inclusion either $C \subset D$ or $D \subset C$ and in either case we see that there is one set in $\mathcal{R}$ which contains both $a$ and $b$. Since each set in $\mathcal{R}$ is a chain ordered subset of $P$ it follows that either $a \preccurlyeq b$ or $b \preccurlyeq a$. This proves that $A \in \mathcal{T}$ and it is then easy to see that $A=\sup \mathcal{R}$. Thus $\mathcal{T}$ satisfies the condition of chain completeness of (d). By definition of $f$ we have $A \subset f(A)$. Also condition (ii) of (d) is satisfied. Also, it follows immediately that condition (iii) of (d) is satisfied.

We can now conclude from (d) that there are sets $A_{k} \in \mathcal{T}$ with $f\left(A_{k}\right)=$ $A_{k}$ for $k \in \mathbb{N}$ or $f$ has finite fixed points. Thus we have a contradiction. The proof is now complete.

Theorem 7. Let $P:=(P, \preccurlyeq)$ be a partially ordered set. Then the following statements are equivalent:

(a) (Lemma od Infinite Maximalily). Let $P$ be quasi inductive partially ordered set, then $P$ has at least countable maximal elements or $P$ has at least finite maximal elements.

(b) Let $P$ be a quasi inductive partially ordered set and $f$ a mapping from $P$ into itself such that the following fact holds in the form as

$$
x \preccurlyeq f^{2}(x) \text { for all } x \in \operatorname{Sub} f(P),
$$

then $f$ has at least countable fixed apices or $f$ has at least finite fixed apices.

The proof of this statement is very similar with the preceding proof of Theorem 4. Thus the proof of this statement we omit.

\section{Zermelo's Axiom of Choice}

Theorem 8. (Axiom of Choice for Points). Let $P$ be a set partially ordered by an ordering relation $\preccurlyeq$. Then the following statements are equivalent:

(a) (Zorn's lemma). Let $P$ be an inductive partially ordered set. Then $P$ has at least one maximal element. 
(b) Let $P$ be an inductive partially ordered set and $f$ a mapping from $P$ into $P$ such that

$$
x \preccurlyeq \sup \{x, f(x)\} \quad \text { for all } \quad x \in \operatorname{Sub} f(P),
$$

then the function $\varphi(x):=\sup \{x, f(x)\}$ has at least one fixed point. If for all $a, b \in P$ the following condition holds in the form as

$$
\sup \{a, b\}=a \quad \text { implies } \quad a=b,
$$

then the mapping $f$ has at least one fixed point. (Also, the dually statement of this statement holds).

(c) Let $P$ be an inductive partially ordered set and $f$ a mapping from $P$ into $P$ such that

$$
x \preccurlyeq f(x) \text { for all } x \in \operatorname{Sub} f(P),
$$

then $f$ has at least one fixed point. (Also, the dually statement of this statement holds).

Proof. From Tasković [23, Theorem 1, p. 898], (a) is equivalent to the (c). Thus, we need only show that (a) implies (b) and that (b) implies (c).

(a) implies (b). By Zorn's lemma there exists a maximal element $z \in P$, i.e., Sub $f(P)$ has a maximal element $z \in \operatorname{Sub} f(P)$. From condition (M) we have $z \preccurlyeq \sup \{z, f(z)\}=z$, and, because $z$ is a maximal element of set Sub $f(P), \sup \{z, f(z)\} \preccurlyeq z$. Hence, $\varphi(z):=\sup \{z, f(z)\}=z$, i.e., $\varphi$ has a fixed point. Applying condition (A) to the equality $\varphi(z)=z$ we obtain $f(z)=z$. Thus $f$ has fixed point.

(b) implies (c). From (N) we have $x \preccurlyeq f(x) \preccurlyeq \sup \{x, f(x)\}$ for all $x \in \operatorname{Sub} f(P)$. Since (M) holds, it follows from (b) that $\varphi(x):=\sup \{x, f(x)\}$ has a fixed point $\xi \in \operatorname{Sub} f(P)$. Thus, and from (N), the condition (A) holds for the points $\xi$ and $f(\xi)$. Applying (b) to this fact we obtain that $f$ has a fixed point. The proof is complete.

Annotations. The proof that (c) implies (a) may be found on Tasković [23, p. 898] and on Tasković $[20$, p. 85]. In connection with this we give in the next a different proof of the preceding:

(c) implies (a). (Proof sketch). Suppose that (a) is false. Let $L_{0}$ be the family of all nonempty linearly ordered subsets of $P$ and let $L=L_{0} \cup\{\varnothing\}$. The family $L$ is partially ordered by the inclusion relation $\subset$ between subsets of $P$. For each $A \in L_{0}$ the set

$$
A_{U}=\{x \in P: x \text { is an upper bound for } A \text { and } x \notin A\}
$$

is nonempty because if $x$ is an upper bound for $A$ and $y \in P$ is such that $x \preccurlyeq y$ and $x \neq y$ then $u \in U_{A}$. Let $U_{\varnothing}=\left\{x_{0}\right\}$, where $x_{0}$ is an arbitrary element of $P$.

Let $f$ be a function defined on $L$ such that $f(A)=A \cup\left\{g\left(U_{A}\right)\right\}$ for all $A \in L$, where $g$ is the identity mapping $g(x)=x$ with domain $\left\{U_{A}: A \in L\right\}$. 
Now, $L$ satisfies the condition of inductiveness of (c). By definition of $f$ we have $A \subset f(A)$ so that condition (N) of (c) is satisfied; but $f$ has no fixed point, i.e., we have a contradiction. This completes the proof.

In connection with the preceding statement, we notice that statement (b) of Theorem 8 we can give in the following form: Let $P$ be an inductive poset and $f$ a mapping from $P$ into itself such that $(\mathrm{M})$, then the equation in the form as

$$
\sup \{x, f(x)\}=x
$$

has at least one solution on the set $P$. If condition (A) holds, then the equation $x=f(x)$ has at least one solution on the set $P$.

Theorem 9. (Axiom of Choice for Apices). Let $P$ be a set partially ordered by an ordering relation $\preccurlyeq$. Then the following statements are equivalent:

(a) (Zorn's lemma). Let $P$ be an inductive partially ordered set. Then $P$ has at least one maximal element.

(b) Let $P$ be an inductive partially ordered set and $f$ a mapping from $P$ into $P$ such that

$$
x \preccurlyeq \sup \left\{x, f^{2}(x)\right\} \text { for all } x \in \operatorname{Sub} f(P),
$$

then the function $\psi(x):=\sup \left\{x, f^{2}(x)\right\}$ has at least one fixed point. If condition (A) holds, then the mapping $f$ has a fixed apex. (Also, the dually statement of this statement holds).

(c) Let $P$ be an inductive partially ordered set and $f$ a mapping from $P$ into $P$ such that

$$
x \preccurlyeq f^{2}(x) \text { for all } x \in \operatorname{Sub} f(P),
$$

then $f$ has at least one fixed apex. (Also, the dually statement of this statement holds).

Proof. From Tasković [23, Theorem 1, p. 898], (a) is equivalent to the (c). Thus, we need only show that (a) implies (b) and that (b) implies (c). First, (a) implies (b). By Zorn's lemma there exists a maximal element $z \in P$, i.e., Sub $f(P)$ has a maximal element $z \in \operatorname{Sub} f(P)$. From condition (R) we have $z \preccurlyeq \sup \left\{z, f^{2}(z)\right\}$ and, because $z$ is a maximal element of set Sub $f(P), \sup \left\{z, f^{2}(z)\right\} \preccurlyeq z$. Hence, $\psi(z):=\sup \left\{z, f^{2}(z)\right\}=z$, i.e., $\psi$ has a fixed point. Applying condition (A) to the equality $\psi(z)=z$ we obtain $f^{2}(z)=z$. This means, from the initial facts for apices, that $f$ has fixed apices $z, f(z) \in P$ because $z=f^{2}(z)$ and $f(z)=f(z)$.

(b) implies (c). From (T) we have $x \preccurlyeq f^{2}(x) \preccurlyeq \sup \left\{x, f^{2}(x)\right\}$ for all $x \in$ Sub $f(P)$. Since $(\mathrm{R})$ holds, it follows from (b) that $\psi(x):=\sup \left\{x, f^{2}(x)\right\}$ has a fixed point $\eta \in \operatorname{Sub} f(P)$. Thus, and from (T), the condition (A) holds for the points $\eta$ and $f^{2}(\eta)$. Applying (b) to this fact we obtain that $f$ has a fixed apex. The proof is complete. 
Annotations. In connection with the preceding two statements we notice that as proved in Tasković [23] the following statements are equivalent: Zorn's lemma and the following statement.

Also, we notice that statement (b) of Theorem 9 we can give in the following profitable form for applications: Let $P$ be an inductive poset and $f$ a mapping from $P$ into itself such that $(\mathrm{R})$, then the equation in the form as

$$
\sup \left\{x, f^{2}(x)\right\}=x
$$

has at least one solution on the set $P$. If condition (A) holds, then the equation $x=f^{2}(x)$ has at least one solution on the set $P$.

Theorem 10. (Zermelo [29]). Let $P$ be a chain complete partially ordered set and $f$ a mapping from $P$ into itself such that: (a) there is an element $\theta \in P$ with $\theta \preccurlyeq x$ for all $x \in P$, (b) $x \preccurlyeq f(x)$ for all $x \in P$, (c) if $x, y \in P$ and $x \preccurlyeq y \preccurlyeq f(x)$ then either $x=y$ or $f(x) \preccurlyeq f(y)$. Then $f$ has a fixed point.

A proof that Zorn's lemma is equivalent to Theorem 10 may be found first time in the author's Thesis [20]. For further facts of this see Tasković [28].

Our next purpose is to prove some results about partial order relations, now usually called Zorn's lemma, which has far-reaching consequences in several branches of mathematics. We give a strong form of Zorn's lemma due to Bourbaki, for well-ordered $(:=$ wo) chains.

Lemma 1. (Bourbaki [4]). Let $P$ be a quasi-inductive partially ordered set. Then $P$ has at least one maximal element.

Let $P$ be a partially ordered set and $f$ a mapping from $P$ into $P$. For any $f: P \rightarrow P$ it is natural to consider the following set of the form as

$$
\operatorname{Sub}[f(P)):=f(P) \cup\{a \in P: a=u b C \text { for some wo chain } C \text { in } f(P)\} \text {, }
$$

where $u b C$ is an upper bound of well-ordered chain in $C$. This set is first time considered in Tasković [23].

In the next, we consider elements of the apices equation for $x \in P$ in the following form

$$
\sup \left\{x, f^{2}(x)\right\}=x
$$

for a given map $f \mid P$ and for a nonempty partially ordered set $P$. Also, this is a key object for new equivalents of the Axiom of Choice.

Lemma 2. (Lemma of Equation Apices). Let $P$ be a quasi-inductive partially ordered set and $f$ a mapping from $P$ into $P$ such that

$$
x \preccurlyeq \sup \left\{x, f^{2}(x)\right\} \text { for all } x \in \operatorname{Sub}[f(P)) \text {, }
$$

then the function $\psi(x):=\sup \left\{x, f^{2}(x)\right\}$ has a fixed point. If the condition (A) holds, then the mapping $f$ has a fixed apex. (Also, the dually statement of this statement holds). 
Proof. By Lemma 1 there exists a maximal element $z \in P$, i.e., $\operatorname{Sub}[f(P))$ has a maximal element $z \in \operatorname{Sub}[f(P))$. From condition (Rw) we have $z \preccurlyeq \sup \left\{z, f^{2}(z)\right\}$ and, because $z$ is a maximal element of $\operatorname{Sub}[f(P))$, we have $\sup \left\{z, f^{2}(z)\right\} \preccurlyeq z$. Hence, $\psi(z)=z$; so applying condition (A) to this fact, from the initial facts for apices, we obtain that $f$ has a fixed apex. The proof is complete.

The lemmas help to obtain the following result. We show later that the existence of a fixed apex for each mapping from a poset into itself that satisfies $(\mathrm{Rw})$ is a necessary and sufficient condition for the poset to be quasi-inductive.

Theorem 11. If $P$ is a poset such that (A) and that every $f: P \rightarrow P$ that satisfies $(\mathrm{Rw})$ has a fixed apex, then $P$ is quasi-inductive. (The Axiom of Choice is not used in this proof).

Proof. We have to show that under the assumption that the poset $P$ is not quasi-inductive there exists a mapping $f$ on $P$ to $P$ with condition $(\mathrm{Rw})$ and without fixed apices.

Suppose $P$ is not quasi-inductive. Then there exists a well-ordered chain $C$ in $P$ that has no upper bound. Let $U$ be a chain cofinal with $C$ such that

$$
U:=\left\{x \in C \mid x_{0} \preccurlyeq x\right\}, \quad x_{0}:=\text { a fixed element of } C=\min U .
$$

Thus all elements of $U$ can be arranged in a sequence, i.e., one can show that there exists a strictly increasing sequence $\left\{x_{\alpha}\right\}$ in $U$ such that its upper bound does not exist. We define a mapping $f$ from $P$ into itself by

$$
f(x)=\left\{\begin{array}{l}
x_{\alpha+1} \text { if } x=x_{\alpha} \in U, \\
x_{0}:=\min U, \text { if } x \notin U,
\end{array}\right.
$$

where $x_{\alpha} \preccurlyeq x_{\alpha+1}\left(x_{\alpha} \neq x_{\alpha+1}\right)$ for any $\alpha \prec w$, and where $w$ is any ordinal. Now, for any $x \in U(\supset \operatorname{Sub}[f(P)))$ we have $x \preccurlyeq f^{2}(x) \preccurlyeq \sup \left\{x, f^{2}(x)\right\}$, i.e., $x=x_{\alpha} \preccurlyeq x_{\alpha+2}=f\left(x_{\alpha+1}\right)=f\left(f\left(x_{\alpha}\right)\right)=f^{2}\left(x_{\alpha}\right)=f^{2}(x) \preccurlyeq \sup \left\{x, f^{2}(x)\right\}$ for $\alpha \prec w$, so $f$ satisfies (Rw) and does not have a fixed apex. The proof is complete.

The following result of Bourbaki [4] allows us to prove the basic fixpoint statement for chain complete posets. Also see and an essential result by Amann [3].

Lemma 3. Let $P$ be a chain complete partially ordered set and $f: P \rightarrow P$ a map such that $x \preccurlyeq f(x)$ for all $x \in P$. Then $f$ has a fixed point.

Our next statement extends this lemma with chain complete posets to quasi-inductive partially ordered sets in the following sense.

Lemma 4. (Lemma of Equation Points). Let $P$ be a quasi-inductive partially ordered set and $f: P \rightarrow P$ a map such that

$$
x \preccurlyeq \sup \{x, f(x)\} \text { for all } x \in \operatorname{Sub}[f(P)) \text {, }
$$


then the function $\varphi(x):=\sup \{x, f(x)\}$ has at least one fixed point. If condition (A) holds, then the mapping $f$ has a fixed point. (Also, the dually statement of this statement holds).

The proof of this statement is a totally analogous with the proof of Lemma 2. A brief proof of this statement based on Bourbaki's lemma may be found in Tasković [23].

We say that $P$ has the equation-general fixed point property if (A) and if every map $f$ of $P$ into itself with condition $(\mathrm{Mw})$ has a fixed point. Analogously, $P$ is said to have the equation-general fixed apex property if (A) and if every map $f$ of $P$ into itself with condition $(\mathrm{Rw})$ has a fixed apex.

Theorem 12. Let $P$ be a partially ordered set. Then the following statements are equivalent:

(a) $P$ is quasi-inductive,

(b) $P$ has the equation-general fixed apex property,

(c) $P$ has the equation-general fixed point property.

Proof. From Theorem 11 and Lemma 2, (a) is equivalent to (b). Lemma 4 implies that (c) is a consequence of (a). Thus, we need only show that (c) implies (a).

Suppose $P$ is not quasi-inductive. Then there is a chain $C$ in $P$ that does not have an upper bound. Let $U$ be an ordered chain cofinal with $C$. Define a mapping $f$ from $P$ into itself by (3). Then $f$ is well defined and for any $x \in \operatorname{Sub}[f(P))$ we have $x \preccurlyeq f(x) \preccurlyeq \sup \{x, f(x)\}$, i.e., $x=x_{\alpha} \preccurlyeq x_{\alpha+1}=$ $f\left(x_{\alpha}\right)=f(x) \preccurlyeq \sup \{x, f(x)\}$. Thus, $f$ satisfies (Mw) and does not have a fixed point.

Theorem 13. If $P$ is a poset such that (A) and that every mapping $f: P \rightarrow$ $P$ that satisfies $(\mathrm{R})$ has a fixed apex, then $P$ is quasi-inductive. (The Axiom of Choice is not used in the proof).

The proof of this statement is analogous to the proof of the preceding statements. A brief similarly proof of this statement may be found in Tasković [28].

As indicated in Tasković [28], $P$ is said to have the equation fixed point property if (A) and if every map $f$ of $P$ into itself with the condition (M) has a fixed point. Analogously, $P$ is said to have the equation fixed apex property if (A) and if every map $f$ of $P$ into itself with the condition (R) has a fixed apex. The following statement summarizes things in Tasković [23] and in preceding statements.

Theorem 14. Let $P$ be a partially ordered set. Then the following statements are equivalent: (a) $P$ is inductive, (b) $P$ is quasi-inductive, (c) $P$ has the equation fixed apex property, (d) $P$ has the equation fixed point property, (e) $P$ has the equation-general fixed apex property, (f) $P$ has the equationgeneral fixed point property. 
We notice, as an immediate application of the preceding statements (equivalents of inductiveness and quasi-inductiveness), that Zorn's lemma and Bourlaki's lemma are equivalent. Analogously, Bourbaki's lemma, Lemma of Equation Apices, and Lemma of Equation Points are equivalent. Also, we have the following statement as a consequence.

Let $P$ be a partially ordered set and $f$ a mapping from $P$ into $P$. For any $f: P \rightarrow P$ it is natural to consider the set

$$
\overline{f(P)}:=f(P) \cup\{a \in P \mid a=\sup C \text { for some chain } C \text { in } f(P)\} .
$$

Theorem 15. Let $P$ be a set partially ordered by an ordering relation $\preccurlyeq$. Then the following statements are equivalent:

(a) (Bourbaki's lemma). Let $P$ be a quasi-inductive partially ordered set, then $P$ has at least one maximal element.

(b) Let $P$ be a quasi-chain complete partially ordered set. Then $P$ has at least one maximal element.

(c) Let $P$ be a chain complete partially ordered set and $f$ a mapping from $P$ into itself such that

$$
x \preccurlyeq \sup \left\{x, f^{2}(x)\right\} \text { for all } x \in \overline{f(P)},
$$

then the function $\psi(x):=\sup \left\{x, f^{2}(x)\right\}$ has a fixed point. If the condition (A) holds, then the mapping $f$ has a fixed apex. (Also, the dually statement of this statement holds).

(d) Let $P$ be a chain complete partially ordered set and $f$ a mapping from $P$ into itself such that

$$
x \preccurlyeq \sup \{x, f(x)\} \text { for all } x \in \overline{f(P)},
$$

then the function $\varphi:=\sup \{x, f(x)\}$ has a fixed point. If the condition (A) holds, then the mapping $f$ has a fixed point. (Also, the dually statement of this statement holds).

Based on Theorem 15 the above facts, we can prove the following statement as a localization of the preceding statement.

Theorem 16. (Local forms). Let $P$ be a partially ordered set with ordering relation $\preccurlyeq$. Then the following statements are equivalent:

(a) (Zorn's lemma). Let $P$ be an inductive partially ordered set. Then $P$ has at least one maximal element.

(b) Let $P$ be a quasi-chain complete partially ordered set and $f$ an increasing mapping from $P$ into $P$ such that

$$
a \preccurlyeq \sup \left\{a, f^{2}(a)\right\} \text { for some } a \in P,
$$

then the function $\psi(x):=\sup \left\{x, f^{2}(x)\right\}$ has a fixed point. If the condition (A) holds, then the mapping $f$ has a fixed apex. (Also, the dually statement of this statement holds). 
(c) Let $P$ be a quasi-chain complete partially ordered set and $f$ an increasing mapping from $P$ into $P$ such that

$$
a \preccurlyeq \sup \{a, f(a)\} \text { for some } a \in P,
$$

then the function $\varphi(x):=\sup \{x, f(x)\}$ has a fixed point. If the condition (A) holds, then the mapping $f$ has a fixed point. (Also, the dually statement of this statement holds).

Proof. (a) implies (b). Consider the subset $A$ of $P$ given by $A:=$ $\left\{x \in P: x \preccurlyeq \sup \left\{x, f^{2}(x)\right\}\right.$. From the given condition we see that $A$ is nonempty. Since $x \preccurlyeq \sup \left\{x, f^{2}(x)\right\}$ implies $f(x) \preccurlyeq \sup \left\{f(x), f^{2}(f(x))\right\}$, and we therefore have that $f$ maps $A$ into $A$. Next, let $C$ be a nonempty well-ordered chain of $A$ and $\xi$ a least upper bound of $C$. Also, since $x \preccurlyeq \xi$ for every $x \in C$, it follows that $f^{2}(x) \preccurlyeq f^{2}(\xi)$ for every $x \in C$. However, since $C$ is a subset of $A$, it follows that $x \preccurlyeq \sup \left\{x, f^{2}(x)\right\} \preccurlyeq \sup \left\{\xi, f^{2}(\xi)\right\}$ for every $x \in C$. So $\sup \left\{\xi, f^{2}(\xi)\right\}$ is an upper bound of $C$ and, consequently, $\xi \preccurlyeq \sup \left\{\xi, f^{2}(\xi)\right\}$. It then follows that $A$ is a nonempty poset with the property that each nonempty well-ordered chain of $A$ has an upper bound, i.e., $A$ is a quasi-inductive set and $f$ maps $A$ into $A$; thus, according to (b) of Theorem 9, we see that $f$ has a fixed apex, as described.

The proofs for (b) implies (c) and (c) implies (a) are analogous to the proofs of the statements (b) implies (c) and (c) implies (a) of Theorems 8 and 9 .

An immediate consequence of the preceding Lemma of Equation Points and its proof is the following equivalent form of the Axiom of Choice.

Theorem 17. Let $\mathcal{F}$ be a family of mappings of a partially ordered set $P$ into itself with ordering $\preccurlyeq$ such that

$$
x \preccurlyeq \sup \{x, f(x)\} \text { for all } x \in P \text {, all } f \in \mathcal{F} ;
$$

and if $P$ is a quasi-inductive poset with the condition (A), then the family $\mathcal{F}$ has a common fixed point for all $f \in \mathcal{F}$.

On the other hand, an immediate consequence of the above Lemma of Equation Apices and its proof is the following equivalent form of the Axiom of Choice, also.

Theorem 18. Let $\mathcal{F}$ be a family of mappings of a partially ordered set $P$ into itself with ordering $\preccurlyeq$ such that

$$
x \preccurlyeq \sup \left\{x, f^{2}(x)\right\} \text { for all } x \in P \text {, all } f \in \mathcal{F} \text {; }
$$

and if $P$ is a quasi-inductive poset with the condition (A), then the family $\mathcal{F}$ has a common fixed apex for all $f \in \mathcal{F}$.

The proof of this statement is analogous to the proof of the preceding statement. A brief proof a similarly statement may be found in Tasković [23]. 
Annotations. In concluding, we note that the fixed apex theorems can easily be generalized from $f^{2}$ to $f^{n}:=f\left(f^{n-1}\right)$ for $n \geq 2$.

The conception of apices can be broadened from $f^{2}$ to $f^{n}$ for some positive integer $n \geq 2$. The points $u_{1}, \ldots, u_{n} \in P$ are called fixed apices of $f: P \rightarrow P$ if

$$
u_{1}=f\left(u_{2}\right), \quad u_{2}=f\left(u_{3}\right), \ldots, u_{n-1}=f\left(u_{n}\right), \quad u_{n}=f\left(u_{1}\right)
$$

or if

$$
u_{2}=f\left(u_{1}\right), \quad u_{3}=f\left(u_{2}\right), \ldots, u_{n}=f\left(u_{n-1}\right), \quad u_{1}=f\left(u_{n}\right)
$$

for some positive integer $n \geq 2$. For this conception of apices also see Tasković [23].

We notice that the map $f$ has fixed apices $u_{1}, \ldots, u_{n} \in P$ if and only if $f^{n}$ (for some $n \geq 2$ ) has a fixed point.

Indeed, for if $f$ has fixed apices $u_{1}, \ldots, u_{n} \in P$ then $u_{1}=f\left(u_{2}\right)=$ $f^{2}\left(u_{3}\right)=\cdots=f^{n}\left(u_{1}\right)$ or $u_{n}=f^{n}\left(u_{1}\right)=f\left(u_{n-1}\right)=\cdots=f^{n-1}\left(u_{1}\right)=$ $f^{n}\left(u_{n}\right)$, so $f^{n}$ has a fixed point. On the other hand, if the equation $x=f^{n}(x)$ has a solution $\xi=f^{n}(\xi)$ for some $\xi \in P$, then $f$ has a fixed apices $\xi, f^{n-1}(\xi), f^{n-2}(\xi), \cdots, f(\xi)$ because $\xi=f^{n}(\xi), f^{n-1}(\xi)=f\left(f^{n-2}(\xi)\right), \cdots$, $f(\xi)=f(\xi)$.

It will be observed that all results for fixed apices from this paper as and from Tasković [23] hold for the generalizations of fixed apices given by $(\mathrm{AL})$ and $(\mathrm{AR})$. We state only the following basic result, which is also an equivalent form of the Axiom of Choice.

Lemma 5. Let $P$ be a quasi-inductive partially ordered set with ordering $\preccurlyeq$ and $f$ a mapping from $P$ into $P$ such that for some positive integer $n \geq 2$ is

$$
x \preccurlyeq \sup \left\{x, f^{n}(x)\right\} \text { for all } x \in \operatorname{Sub}[f(P)),
$$

then the function $\alpha(x):=\sup \left\{x, f^{n}(x)\right\}$ has a fixed point. If condition (A) holds, then the mapping $f$ has fixed apices. (Also, the dually statement of this statement holds).

The proof of this statement is analogous to the proof of Lemma 2. A brief proof of this statement for a similarly result may be found in Tasković [28].

Annotation. In connection with the preceding statement, we notice that Lemma 5 we can give in the following profitable form for applications: Let $P$ be a quasi-inductive poset and $f$ a mapping from $P$ into itself such that for some positive integer $n \geq 2$ is (Ra), then the equation in the form as

$$
\sup \left\{x, f^{n}(x)\right\}=x
$$

has at least one solution on the set P. If condition (A) holds, then the equation $x=f^{n}(x)$ has at least one solution on the set $P$. 


\section{Foundation of the Fixed Point Theory}

We notice that, de facto, Lemma od Equation Points, i.e., Axiom of Choice is main geometrical spring for the Fixed Point Theory. In this sense we give some illustrations of equations (Eq), (Aq), statement (b) of Theorem 8, statement (b) of Theorem 9, and the following equation for $x \in P$ and for some positive integer $n \geq 2$ in the form as

$$
\sup \left\{x, f^{n}(x)\right\}=x
$$

for a given map $f \mid P$ and for a nonempty partially ordered set $P$. This is also general a key object for new equivalents of the Axiom of Choice.

Illustration 1. (Tarski's theorem). Let $P$ be a poset. A self-mapping $f$ of $P$ into itself is called an isotone mapping if $x \preccurlyeq y$ implies $f(x) \preccurlyeq f(y)$. In the mid-1950's A. Tarski published a generalization of a former fixed point theorem for posets goes back to Tarski and Knaster in the form: Every complete lattice has the fixed point property, i.e., every isotone map $f \mid P$ of a complete lattice $P$ into itself has a fixed point. The Axiom of Choice is not used in the Tarski's proof.

But, we notice that Tarski's theorem is a consequence of the Axiom of Choice. Indeed, if for a complete lattice $P$ holds the Tarski's theorem, then $P$ is an inductive poset and the condition (M) of (b) in Theorem 8 holds. Since condition (A) also holds on complete lattice $P$ in the form that for an arbitrary isotone mapping $f: P \rightarrow P$ there exists an element $x \in P$ such that

$$
\sup \{x, f(x)\}=x \text { implies } x=f(x),
$$

hence applying statement (b) of Theorem 8 (i.e., Axiom of Choice) we obtain that $f$ has a fixed point. The proof is complete.

Annotations. From the preceding illustration we have that Tarski's theorem is a consequence of Axiom of Choice. This is a result of the fact that the equation for $x \in P$ and for given an arbitrary isotone mapping $f: P \rightarrow P$ in the form as

$$
\sup \{x, f(x)\}=x
$$

has at least one solution on a complete lattice $P$. But, very interesting, for given an arbitrary antitone mapping $f: P \rightarrow P$ the equation (Fp) has not always solution on a complete lattice $P$. For this see the following fact.

Illustration 2. (The equation $\sup \left\{x, f^{2}(x)\right\}=x$ ). Let $P$ be a poset. A self-mapping $f$ on $P$ into itself is called an antitone mapping if $x \preccurlyeq y$ implies $f(y) \preccurlyeq f(x)$. In Tasković [21] we have without the Axiom of Choice the following result of J. Klimeš: If $P$ is a complete lattice and if $f$ is an antitone mapping of $P$ into itself, then $f$ has a fixed apex.

We notice that this result of Klimeš [9] is a consequence of the Axiom of Choice. Indeed, if for a complete lattice $P$ holds the preceding result, then $P$ is an inductive poset and the condition $(\mathrm{R})$ of $(\mathrm{b})$ in Theorem 9 holds. 
Since in this case, from Illustration 1, the condition (A) holds because the equation in the form (Aq) has a solution, thus applying statement (b) of Theorem 9 (i.e., Axiom of Choice) we obtain that $f$ has a fixed apex. The proof is complete.

Illustration 3. (Brouwer's theorem). The most famous of all fixed point theorems is that of Brouwer which satisfies that: every continuous mapping of the closed unit ball in the Euclidean space $\mathbb{R}^{n}$ into itself has a fixed point.

A new proof for this result (without Axiom of Choice) may be found in Tasković [28]. But, we notice that Brouwer's theorem is a consequence of the Axiom of Choice, also.

Indeed, if $\leq$ well-ordered on $P:=\mathbb{R}^{n}$ (from the well-ordering theorem), then every chain in $P$ has a lower bound and dually condition of $(\mathrm{M})$ for (b) in Theorem 8 holds. Since the dually condition of (A) also holds, thus applying the dually statement of (b) in Theorem 8 (i.e., Axiom of Choice) we obtain that $f$ has a fixed point. The proof is complete.

Annotations. In the preceding context of this illustration we obtain the following result: If $C$ is a nonempty convex compact subset of $\mathbb{R}^{n}$ and if $f: C \rightarrow C$ is a continuous mapping, then the equation (Fp) has a solution on $C$.

Illustration 4. (Schauder's theorem). Schauder's theorem is a generalization of Brouwer's theorem to infinite dimensional normed linear spaces. Schauder's theorem states that: every continuous mapping of a compact convex subset of a normed linear space into itself has a fixed point.

Also, Schauder's theorem is a consequence of the Axiom of Choice. Indeed, in the same manner as in the preceding illustration we have the following result: If $C$ is a nonempty compact convex subset of a Banach space and if $f: C \rightarrow C$ is a continuous operator, then the equation $(\mathrm{Fp})$ has a solution on $C$. Thus, we have and Schauder's theorem as a consequence of the fact (b) in Theorem 8, i.e., as a consequence of the Axiom of Choice.

Illustration 5. (Schauder's problem). The most famous of many problems in nonlinear analysis is Schauder's problem (Scottish book, problem 54) of the following form, that if $C$ is a nonempty convex compact subset of a linear topological space does every continuous mapping $f: C \rightarrow C$ has a fixed point? The answer we give in Tasković [28] is yes.

Theorem 19. (Answer is yes for Schauder's problem). Let $C$ be a nonempy convex compact subset of a linear topological space $X$ and suppose $T: C \rightarrow C$ is a continuous mapping. Then $T$ has a fixed point in $C$.

The proof of this fact may be found in Taskovic [28] by the aid of the Axiom of Choice, i.e., of Zorn's lemma. On the other hand, a proof that solution of Schauder's problem as Theorem 19 is a consequence of Lemma of Equation Points we can give in the same manner as the proof of Brouwer's theorem. 
In this case also, de facto, we give that: if $C$ is a nonempty convex compact subset of a linear topological space and if $f: C \rightarrow C$ is a continuous mapping, then the equation (Fp) has a solution on $C$.

Illustration 6. (Caristi's theorem). In $1976 \mathrm{~J}$. Caristi investigated a mapping $f$ on a complete metric space $(X, \rho)$ that satisfies the following condition: there exists a lower semicontinuous function $G: X \rightarrow \mathbb{R}_{+}^{0}:=$ $[0,+\infty)$ such that

$$
\rho[x, f(x)] \leq G(x)-G(f(x)) \text { for every } x \in X,
$$

and showed that such mappings have a fixed point in space $X$. In this sense, define a relation $\preccurlyeq_{G, \rho}$ on $X$ by the following condition in the form as:

$$
a \preccurlyeq \rho, G b \text { if and only if } \rho[a, b] \leq G(a)-G(b) \text {; }
$$

it is verify that $\preccurlyeq \rho, G$ is a partial ordering (asymmetric and transitive relation) in $X$. Via this ordering Kirk [Colloq. Math., 36 (1976), 81-86] give fact that Zorn's lemma implies Caristi's theorem.

On the other hand, a different proof that Caristi's theorem is a consequence of Lemma of Equation Points, i.e., of the Axiom of Choice we can give in the same manner as the proof of Brouwer's theorem via ordering $\preccurlyeq G, \rho$. For a different proof for this fact see: Browder [Seminar of Fixed Point Theory and its Appl., Dalhousie University, June 1975, 23-27].

Illustration 7. (Bounds roots of equations). We notice that, by the application by Tasković [28, Lemma 2, p. 241] one can simultaneously obtain the upper and lower bounds of the roots of the following equation in the form

$$
\begin{gathered}
x^{n}=a_{1} x^{n-1}+a_{2} x^{n-2}+\cdots+a_{n} \\
\left(a_{1}+\cdots+a_{n}>0 ; \quad a_{i} \geq 0(i=1, \ldots, n)\right) .
\end{gathered}
$$

Then, by Minimax Principle in Tasković [28, p. 245], as an immediate consequence we obtain the following statement for solutions of algebraic equations of the preceding form.

Theorem 20. (Tasković $[28]$ ). A point $\xi \in \mathbb{R}_{+}:=(0,+\infty)$ is the root of the equation $(A E)$ if and only if the following equality holds

$$
\begin{aligned}
& \xi:=\max _{\lambda_{2}, \ldots, \lambda_{n} \in \mathbb{R}_{+}} \min \left\{\lambda_{2}, \ldots, \lambda_{n}, a_{1}+\frac{a_{2}}{\lambda_{2}}+\cdots+\frac{a_{n}}{\lambda_{n}^{n-1}}\right\}= \\
& =\min _{\lambda_{2}, \ldots, \lambda_{n} \in \mathbb{R}_{+}} \max \left\{\lambda_{2}, \ldots, \lambda_{n}, a_{1}+\frac{a_{2}}{\lambda_{2}}+\cdots+\frac{a_{n}}{\lambda_{n}^{n-1}}\right\} .
\end{aligned}
$$

We notice that, if $0<a_{1}+\cdots+a_{n}<1$, then a root $\xi$ of the equation (AE) of the form (Ro) lie in the open interval $(0,1)$, i.e., $\xi \in(0,1)$, such 
that

$$
\begin{aligned}
\xi & =\max _{\lambda_{2}, \ldots, \lambda_{n} \in(0,1)} \min \left\{\lambda_{2}, \ldots, \lambda_{n}, a_{1}+\frac{a_{2}}{\lambda_{2}}+\cdots+\frac{a_{n}}{\lambda_{n}^{n-1}}\right\}= \\
& =\min _{\lambda_{2}, \ldots, \lambda_{n} \in(0,1)} \max \left\{\lambda_{2}, \ldots, \lambda_{n}, a_{1}+\frac{a_{2}}{\lambda_{2}}+\cdots+\frac{a_{n}}{\lambda_{n}^{n-1}}\right\} .
\end{aligned}
$$

We notice that this result is, de facto, also a consequence of the Axiom of Choice in the existence sense as a direct consequence of the Axiom of Choice, i.e., of Lemma of Equation Points.

Indeed, for $P:=[0,+\infty]$ with the ordinary ordering $\leq$ we have that $P$ is an inductive set and that in this case for function $f: P \rightarrow P$ defined by $f(x)=a_{1}+a_{2} / x+\cdots+a_{n} / x^{n-1}$ we have that condition (M) of (b) in Theorem 8 trivially holds in the form as

$$
x \leq \max \left\{x, a_{1}+\frac{a_{2}}{x}+\cdots+\frac{a_{n}}{x^{n-1}}\right\} \text { for all } x \in P ;
$$

hence, applying the statement (b) of Theorme 8 (i.e., Axiom of Choice) we obtain that the equation (AE) has a solution on $P$.

Annotation. We notice that the preceding result in above illustration is foundation on the fact that the equation in the form as

$$
\max \left\{x, a_{1}+\frac{a_{2}}{x}+\cdots+\frac{a_{n}}{x^{n-1}}\right\}=x
$$

has at least one solution on the totally ordered set $P:=[0,+\infty]$ because $x \mapsto x$ and $x \mapsto f(x)$ are continuous functions on $P$.

Illustration 8. (Further equivalents Axiom of Choice). In connection with preceding, let $(X, \rho)$ be a metric space and $G: X \rightarrow \mathbb{R}_{+}^{0}:=[0,+\infty)$ be a given function. Define a relation $\preccurlyeq$ on $X$ as Brøndsted ordering by the following condition:

(B) $\quad a \preccurlyeq b$ if and only if $\rho[a, b] \leq G(a)-G(b)$ for all $a, b \in X$.

On the other hand, define a relation $\preccurlyeq G, \rho$ on the metric space $X$ as Tasković ordering by the following condition:

$$
a \preccurlyeq G, \rho b \quad \text { if and only if } \rho[a, b] \leq G(b)-G(a)
$$

for all $a, b, \in X$.

We notice that the ordering (B) is not dually, in comparable, with the ordering (Ta)!

In further, as immediate applications of the preceding Theorems 8 and 9 we have the following results.

In this sense as an immediate consequence of Theorem 9 we obtain by Tasković [28] the following result.

Theorem 21. (Analytic Principles of Choice, Tasković in 1988). Let P be a partially ordered set with an ordered $\preccurlyeq$. Then the following statements are equivalent: 
(a) (Statement (c) of Theorem 9). If $P$ is an inductive set and $f: P \rightarrow P$ such that

$$
x \preccurlyeq f^{2}(x) \text { for all } x \in \operatorname{Sub} f(P),
$$

then $f$ has a fixed apex.

(b) (Tasković in 1993). If $(X, \rho)$ is a complete metric space and $G: X \rightarrow$ $\mathbb{R}_{+}^{0}$ is a lower semicontinuous function, then in the Brøndsted ordering every $f: X \rightarrow X$ satisfying $x \preccurlyeq f^{2}(x)$ for every $x \in X$ has a fixed apex.

(c) (Tasković in 1993). If $(X, \rho)$ is a complete metric space and $G: X \rightarrow \mathbb{R}$ is a bounded above and upper semicontinuous function, then in the ordering $\preccurlyeq_{G, \rho}$ every $f: X \rightarrow X$ satisfying $x \preccurlyeq_{G, \rho} f^{2}(x)$ for every $x \in X$ has a fixed apex.

Short proof. Define relations $\preccurlyeq$ and $\preccurlyeq G, \rho$ on $X$ by (B) and (Ta), and applying Theorem 8 in two direction, directly, we obtain this statement.

On the other hand, in the preceding sense, as an immediate consequence of Theorem 8 we obtain by Tasković [28] the following result.

Theorem 22. (Analytic forms of Axiom of Choice, Tasković in 1988). Let $P$ be a partially ordered set with an ordered $\preccurlyeq$. Then the following statements are equivalent:

(d) (Statement (c) of Theorem 8). If $P$ is an inductive set and $f: P \rightarrow P$ such that

$$
x \preccurlyeq f(x) \quad \text { for all } \quad x \in \operatorname{Sub} f(P),
$$

then $f$ has a fixed point.

(e) (Caristi in 1976). If $(X, \rho)$ is a complete metric space and $G: X \rightarrow \mathbb{R}_{+}^{0}$ is a lower semicontinuous function, then in the Brøndsted ordering every $f: X \rightarrow X$ satisfying $x \preccurlyeq f(x)$ for every $x \in X$ has a fixed point.

(h) (Tasković in 1986 and in 1988). If $(X, \rho)$ is a complete metric space and $G: X \rightarrow \mathbb{R}$ is a bounded above and upper semicontinuous function, then in the ordering $\preccurlyeq_{G, \rho}$ every $f: X \rightarrow X$ satisfying $x \preccurlyeq G, \rho f(x)$ for every $x \in X$ has a fixed point.

Short proof. Define relations $\preccurlyeq$ and $\preccurlyeq G, \rho$ on $X$ by (B) and (Ta), and applying Theorem 8 in two direction, we obtain immediately this statement.

Illustration 9. (Banach's theorem). The notion of order (and the notion of completeness) have each led to fixed point theorems. We now obtain Banach contraction theorem as a consequence of the Axiom of Choice. In this sense let $(X, \rho)$ be a metric space, $T: X \rightarrow X$, and define a relation $\preccurlyeq$ on $X$ by

$$
x \preccurlyeq y \text { if and only if } \rho[x, y] \leq G(x)-G(y)
$$

for all $x, y \in X$, where $G(x)=(1-\alpha)^{-1} \rho[x, T x]$ and $\alpha \in[0,1)$. It is easy to verify that $\preccurlyeq$ is a partial ordering in $X$. Thus $\preccurlyeq:=\preccurlyeq \rho, G$ and we have (as in Illustration 6) that Banach's theorem is a consequence of Lemma of Equation Points, i.e., of the Axiom of Choice. 
Annotation. In connection with the preceding facts from Tasković [28] see and papers: K i r k in 1976, H. H ö ft and P. H ow ard in 1994, A b i a n in 1985, Tur i n i c i in 1984, Brunner in 1987, M a kowski and Wiśniewski in 1969, B aker in 1964, M a ńka in 1988, K e ny o n in 1963, Dj. K u repa in 1952, and B r $\varnothing$ ndsted in 1979.

Illustration 10. (General expansion mappings). Let $X$ be a topological space, $T: X \rightarrow X$, and let $A: X \times X \rightarrow \mathbb{R}_{+}^{0}:=[0,+\infty)$. We shall introduce the concept of CS-convergence in a space $X$; i.e., a topological space $X$ satisfies the condition of CS-convergence iff $\left\{x_{n}\right\}_{n \in \mathbb{N}}$ is a sequence in $X$ and $A\left(x_{n}, T x_{n}\right) \rightarrow 0(n \rightarrow \infty)$ implies that $\left\{x_{n}\right\}_{n \in \mathbb{N}}$ has a convergent subsequence.

Also, we shall introduce the concept of invariant property for space $X$; i.e., a topological space $X$ satisfies the condition of invariant property if there is a nonempty subset $A$ of $X$ such that $T(A)=A$. Obviously, if $T: X \rightarrow X$ is an onto mapping, then $X$ is with the invariant property for $A=X$. Also, if $T: X \rightarrow X$ continuous on a compact space $X$, then $X$ has the invariant property.

Lemma 6. Let the mapping $\varphi: \mathbb{R}_{+} \rightarrow \mathbb{R}_{+}:=(0,+\infty)$ have the following properties in the following form as

$$
\varphi(t)>t \text { and } \liminf _{z \rightarrow t-0} \varphi(z)>t
$$

for every $t \in \mathbb{R}_{+}$. If the sequence $\left\{x_{n}\right\}_{n \in \mathbb{N}}$ of real positive numbers satisfies the inequality of the form as

$$
x_{n} \geq \varphi\left(x_{n+1}\right) \text { for all } n \in \mathbb{N},
$$

then it converges to zero. The velocity of this convergence is not necessarily geometric.

Proof. Since $\left\{x_{n}\right\}_{n \in \mathbb{N}}$ is a nonincreasing bounded sequence in $\mathbb{R}_{+}$, there is a $t \geq 0$ such that $x_{n} \rightarrow t(n \rightarrow \infty)$. We claim that $t=0$. If $t>0$, then

$$
t=\liminf _{n \rightarrow \infty} x_{n} \geq \liminf _{n \rightarrow \infty} \varphi\left(x_{n+1}\right) \geq \liminf _{z \rightarrow t-0} \varphi(z)>t,
$$

which is a contradiction. Consequently $t=0$ and so $x_{n} \rightarrow 0(n \rightarrow \infty)$. The proof is complete.

We are now in a position to formulate the following general statements from: Tasković [28]. Also see: Tasković [19].

Theorem 23. (General expansion). Let $T$ be a mapping of a topological space $X$ into itself, where $X$ with the invariant property and with the condition of CS-convergence. If there is a mapping $\varphi: \mathbb{R}_{+}^{0} \rightarrow \mathbb{R}_{+}^{0}$ such that the condition $(\varphi)$ holds and

$$
A(T x, T y) \geq \varphi(A(x, y)) \text { for all } x, y \in X
$$


where $A: X \times X \rightarrow \mathbb{R}_{+}^{0}, x \mapsto A(x, T x)$ is lower semicontinuous and $A(a, b)=0$ implies $a=b$, then $T$ has a unique fixed point in $X$.

As immediate consequences of the preceding statement we obtain results in 1984 of Wang, Gao, Li and Iséki and in 1992 of Daffer and Kaneko's.

Proof of Theorem 23. (Application of Axiom of Choice). Since $X$ is with invariant property, there exists a nonempty subset $A$ of $X$ such that $T(A)=A$. Then the set of the form $A_{x}:=T^{-1}(\{x\}) \subset A$ is a nonempty subset of $A$ for every $x \in A$. If $g \mid A$ is a function of choice, then there is a sequence $\left\{a_{n}\right\}_{n \in \mathbb{N}}$ in $X$ defined by $a_{n+1}=g\left(A_{a_{n}}\right)$ for $n \in \mathbb{N}$, where $a_{1} \in A$ is an arbitrary point. Thus we obtain that $a_{n}=T\left(a_{n+1}\right)$ for all $n \in \mathbb{N}$, in $X$. From (An) we have

$$
A\left(a_{n}, a_{n+1}\right)=A\left(T\left(a_{n+1}\right), T\left(a_{n+2}\right)\right) \geq \varphi\left(A\left(a_{n+1}, a_{n+2}\right)\right)
$$

for all $n \in \mathbb{N}$. Applying Lemma 6 to the sequence $\left\{A\left(a_{n}, a_{n+1}\right)\right\}_{n \in \mathbb{N}}$, we obtain $A\left(a_{n}, a_{n+1}\right) \rightarrow 0(n \rightarrow \infty)$. This implies (from CS-convergence) that the sequence $\left\{a_{n}\right\}_{n \in \mathbb{N}}$ contains a convergent subsequence $\left\{a_{n(k)}\right\}_{k \in \mathbb{N}}$ with limit $\xi \in X$. Since $x \mapsto A(x, T x)$ is lower semicontinuous at $\xi$, we obtain

$$
A(\xi, T \xi) \leq \liminf A\left(a_{n(k)}, a_{n(k)-1}\right)=\liminf A\left(a_{n}, a_{n-1}\right)=0,
$$

i.e., $T \xi=\xi$. We complete the proof by showing that $T$ can have at most one fixed point. In fact, if $\xi \neq \eta$ were two fixed points, then $A(\xi, \eta)=$ $A(T \xi, T \eta) \geq \varphi(A(\xi, \eta))>A(\xi, \eta)$, which is a contradiction. The proof is complete.

\section{Geometry of the Axiom of Infinite Choice}

The first specific reference to the Axiom of Choice was given in a paper by G. Peano in 1890. In 1892 R. Bettazzi published an article on discontinuous real functions with terminology of infinite many arbitrary choices.

In this sense, we give a demonstration that the equation (Eq) has at least countable solutions or has at least finite solutions. For this see the following figures:
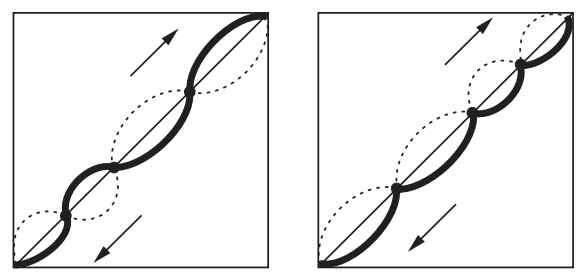

FiguRE 1

Also, as an example, the equation $\sin x=0$ or the equation, in an equivalent form, $x+\sin x=x$. As two extensions of Theorems 8 and 9 on the equation (Eq) we obtain the following two results. 
Theorem 24. (Axiom of Infinite Choice for Points). Let $P$ be a set partially ordered by an ordering relation $\preccurlyeq$. Then the following statements are equivalent:

(a) (Lemma of Infinite Maximality). Let $P$ be an inductive partially ordered set. Then $P$ has at least countable or finite maximal elements.

(b) Let $P$ be an inductive partially ordered set and $f$ a mapping from $P$ into $P$ such that

$$
x \preccurlyeq \sup \{x, f(x)\} \text { for all } x \in \operatorname{Sub} f(P),
$$

then the function $\varphi(x):=\sup \{x, f(x)\}$ has at least countable or finite fixed points. If for all $a, b \in P$ the following condition holds in the form as

$$
\sup \{a, b\}=a \quad \text { implies } \quad a=b,
$$

then the mapping $f$ has at least countable or finite fixed points. (Also, the dually statement of this statement holds).

(c) Let $P$ be an inductive partially ordered set and $f$ a mapping from $P$ into $P$ such that

$$
x \preccurlyeq f(x) \text { for all } x \in \operatorname{Sub} f(P),
$$

then $f$ has at least countable or finite fixed points. (Also, the dually statement of this statement holds).

The proof of this statement is very similar with the preceding proofs. Thus the proof of this statement we omit. Alo and for the next statement!

Theorem 25. (Axiom of Infinite Choice for Apices). Let $P$ be a set partially ordered by an ordering relation $\preccurlyeq$. Then the following statements are equivalent:

(a) (Lemma of Infinite Maximality). Let $P$ be an inductive partially ordered set. Then $P$ has at least countable or finite maximal elements.

(b) Let $P$ be an inductive partially ordered set and $f$ a mapping from $P$ into $P$ such that

$$
x \preccurlyeq \sup \left\{x, f^{2}(x)\right\} \quad \text { for all } \quad x \in \operatorname{Sub} f(P),
$$

then the function $\psi(x):=\sup \left\{x, f^{2}(x)\right\}$ has at least countable or finite fixed points. If condition (A) holds, then the mapping $f$ has at least countable or finite fixed apices. (Also, the dually statement of this statement holds).

(c) Let $P$ be an inductive partially ordered set and $f$ a mapping from $P$ into $P$ such that

$$
x \preccurlyeq f^{2}(x) \text { for all } x \in \operatorname{Sub} f(P),
$$

then $f$ has at least countable or finite fixed apices. (Also, the dually statement of this statement holds).

Essential Facts. We notice that Fredholm and Leray-Schauder alternatives are direct consequences of the Axiom of Infinite Choice. Also, Schauder 
and Brouwer theorem of fixed point (as well as Fixed Point Theory) are direct consequences of the Axiom of Infinite Choice.

In connection with the Axiom of Infinite Choice I have the following direct result as an extension of the Schauder problem (Theorem 19).

Theorem 26. Let $C$ be a nonempty convex compact subset of a linear topological space $X$ and suppose $T: C \rightarrow C$ is a continuous mapping. Then $T$ has at least countable fixed points or $T$ has at least finite fixed points.

Open problem. We notice that in 1963 P. J. Cohen proved that the Axiom of Choice is independent of the remaining Axioms of the Sets Theory. In this sense is the Axiom of Infinite Choice is independent of the remaining Axioms of the Sets Theory? (I think that the answer is yes!?)

Also, in connection with this, I have the following direct result as an extension of the Recursion Theorem.

Theorem 27. If $\xi$ is an element of a set $X$, and if $f$ is a function from $X$ into $X$, then there exist at least countable functions $f_{k}($ for $k \in \mathbb{N})$ or there exist at least finite functions $f_{k}($ for $k=1, \ldots, m$ and a fixed number $m \in \mathbb{N}$ ) from $\mathbb{N}$ into $X$ such that $f_{k}(0)=\xi$ and such that $f_{k}(n \cup\{n\})=f\left(f_{k}(n)\right)$ for all $n \in \mathbb{N}$.

Peano's Theorem. (Initial value problem). Further we give direct an application of Axiom of Infinite Choice to differential equations. As a parallel and contrast to the Picard-Lindelöf theorem we consider the initial value problem of the form as

$$
x^{\prime}(t)=f(t, x(t)), \quad x\left(t_{0}\right)=y_{0},
$$

on $\left[t_{0}-c, t_{0}+c\right]$. Geometrically, (4) means that we are looking for a curve which satisfies the differential equation and passes through $\left(t_{0}, y_{0}\right)$ as in Figure 2 with $y_{0}=p_{0}$. At the end points $t=t_{0} \pm c$, where $x^{\prime}(t)$ is to be interpreted as the appropriate one-sided derivative. The following result is an immediate consequence of the Lemma of Infinite Choice:

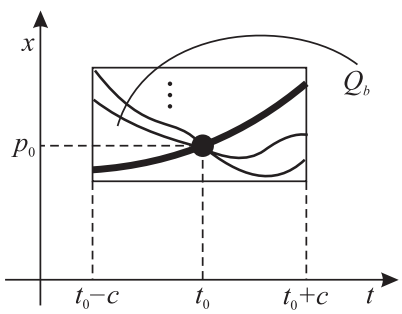

FiguRE 2 
Proposition 1. (Peano [1890], Tasković [2005]). Let there be given real numbers $t_{0}$ and $y_{0}$, and the rectangle of the form as

$$
Q_{b}:=\left\{(t, x) \in \mathbb{R}^{2}:\left|t-t_{0}\right| \leq a,\left|x-y_{0}\right| \leq b\right\},
$$

where $a$ and $b$ are fixed positive numbers. Suppose that $f: Q_{b} \rightarrow \mathbb{R}$ is continuous and bounded with the following condition of the form as

$$
|f(t, x)| \leq K \quad \text { for all }(t, x) \in Q_{b},
$$

and fixed $K>0$. Set $c:=\min \{a, K / b\}$. Then the initial value problem (4) has at least countable or finite continuously differentiable solutions on $\left[t_{0}-c, t_{0}+c\right]$.

\section{REFERENCES}

[1] A. Abian, A fixed point theorem equivalent to the axiom of choice, Abstracts Amer. Math. Soc., abstract no. 388, 4 (1983).

[2] H. Amann, Order structures and fixed points, Ruhr-Universität, Bochum, (mimeographed lecture notes), 1977.

[3] G. Birkhoff, Lattice theory, Amer. Math. Soc. Colloq. Publ., vol. 25, New York, 1948.

[4] N. Bourbaki, Sur le theoreme de Zorn, Archiv der Math. 2 (1950), 434-437.

[5] J. Caristi, Fixed point theorems for mappings satisfying inwardness conditions, Trans. Amer. Math. Soc., 215 (1976), 241-251.

[6] K. Gödel, The consistency of the continuum hypothesis, Ann. of Math. Stud., vol. 3, Princeton Univ. Press, Princeton, NJ, 1940, pp. 66.

[7] P. Howard, and J. E. Rubin, Consequences of the Axiom of Choice, American Math. Society, Vol. 59, 1998. Mathematical Surveys and Monographs, 432 pages.

[8] T. J. Jech, The Axiom of Choice, North-Holland, Amsterdam, 1973.

[9] J. Klimeš, Fixed edge theorems for complete lattices, Arch. Math. 17 (1981), 227 234.

[10] C. Kuratowski, Une methode d'elimination des nombers transfinies raisonnements mathematiques, Fund. Math. 3 (1922), 76-108.

[11] G. Kurepa, Sur la relation d'inclusion et l'axiome de choix de Zermelo, Bull. Soc. Math. France 80 (1952), 225-232.

[12] A. Mostowski, On the principle of dependent choices, Fund. Math. 35 (1948), $127-$ 130 .

[13] G. H. Moore, Zermelo's Axiom of Choice, its origins, development and influence, Springer-Verlag, New York, Heidelberg, and Berlin, Vol. 8 (1982), 410 pages.

[14] H. Rubin, On a problem of Kurepa concerning the axiom of choice, Notices Amer. Math. Soc. 5 (1958), no. 378.

[15] H. Rubin and J. Rubin, Equivalents of the Axiom of Choice, North-Holland, Amsterdam and London, 1970, p. 134.

[16] H. Rubin and J. Rubin, Equivalents of the Axiom of Choice II, North-Holland, Amsterdam, New York and Oxford, 1985, p. 322.

[17] H. Rubin and J. Rubin, Some new forms of the axiom of choice, Notices Amer. Math. Soc. 7 (1960), no. 380.

[18] A. Tarski, A lattice theoretical fixed point theorem and its applications, Pacific J. Math. 5 (1955), 285-309.

[19] M. R. Tasković, Fixed points of contractive and some other mappings, $\mathrm{Ph}$ These, University of Beograd, 1975, 93 pages. 
[20] M. R. Tasković, Banach's mappings of fixed point on spaces and ordered sets, These, Univesity of Beograd, 1978, 151 pages; Math. Balkanica, 9 (1979), 130 pages.

[21] M. R. Tasković, Characterizations of inductive posets with applications, Proc. Amer. Math. Soc. 104 (1988), 650-660.

[22] M. R. Tasković, On an equivalent of the axiom of choice and its applications, Math. Japonica 31 (1986), 979-991.

[23] M. R. Tasković, The axiom of choice, fixed point theorems, and inductive ordered sets, Proc. Amer. Math. Soc., 116 (1992), 897-904.

[24] M. R. Tasković, New maximal principles, Math. Japonica, 37 (1992), 549-554.

[25] M. R. Tasković, Nonlinear Functional Analysis, (Fundamental Elements of Theory). First Book: Monographs, Zavod za udžbenike i nastavna sredstva, Beograd 1993, 812 pages, (Serbo-Croation). English summary: Comments only new main results of this book, Vol. 1 (1993), 713-752.

[26] M. R. Tasković, Nonlinear Functional Analysis, Second Book: Monographs - Global Convex Analysis - General convexity, Variational methods and Optimization, Zavod za udžbenike i nastavna sredstva and Vojnoizdavački zavod, Beograd 2001, (in Serbian), 1223 pages. English summary: Transversal and fixed points, forks, general convex functions, and applications, Vol. 2 (2001), 1067-1176.

[27] M. R. Tasković, Transversal Sets, Math. Moravica, 8-2 (2004), 53-93.

[28] M. R. Tasković, Theory of transversal point, spaces, and forks, Monographs of a new mathematical theory, VIZ-Beograd 2005, (in Serbian), 1054 pages. English summary: 1001-1022.

[29] E. Zermelo, Neuer Beweis fur die Moglichkeit einer Wohlordnung, Math. Ann. 65 (1908), 107-128; translated in van Heijenoort 1967, 183-198.

[30] Max Zorn, A remark on method in transfinite algebra, Bull. Amer. Math. Soc. 41 (1935), 667-670.

Milan R. TASković

Faculty of Mathematics

P.O. Box 550

11000 BEOGRAD

SERBIA

Home address:

Milan R. TAsković

NeHruova 236

11070 BELGRADE

SERBIA

E-mail address: andreja@predrag.us 